\title{
Collaborative \\ Multidisciplinary Design Optimization for Conceptual Design of Complex Products
}

Edris Safavi

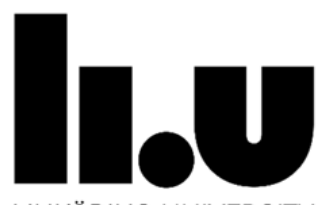

LINKÖPING UNIVERSITY

Division of Machine Design

Department of Management and Engineering

Linköping University, SE-581 83 Linköping, Sweden

Linköping 2016 
Copyright (C) Edris Safavi, 2016

Collaborative Multidisciplinary Design Optimization for Conceptual Design of Complex Products

ISBN: 978-91-7685-712-0

ISSN: 0345-7524

Distributed by:

Division of Machine Design

Department of Management and Engineering

Linköping University

SE-581 83 Linköping, Sweden

Printed in Sweden by LiU-Tryck, Linköping, 2016 
To My Family 
The secret eternal neither you know nor I

And answer to the riddle neither you know nor I

Behind the veil there is much talk about us, why

When the veil falls, neither you remain nor $I$.
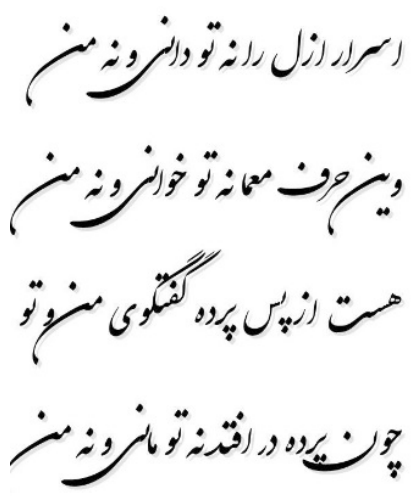

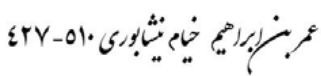

Omar khayam (1048-1122) 


\section{Abstract}

$\mathrm{M}$

ULTIDESCIPLINARY design optimization (MDO) has developed in theory and practice during the last three decades with the aim of optimizing complex products as well as cutting costs and product development time. Despite this development, the implementation of such a method in industry is still a challenge and many complex products suffer time and cost overruns.

Employing higher fidelity models (HFMs) in conceptual design, one of the early and most important phases in the design process, can play an important role in increasing the knowledge base regarding the concept under evaluation. However, design space in the presence of HFMs could significantly be expanded. MDO has proven to be an important tool for searching the design space and finding optimal solutions. This leads to a reduction in the number of design iterations later in the design process, with wiser and more robust decisions made early in the design process to rely on.

In complex products, different systems from a multitude of engineering disciplines have to work tightly together. This stresses the importance of evolving various domain experts in the design process to improve the design from diverse engineering perspectives. Involving more engineers in the design process early on raises the challenges of collaboration, known to be an important barrier to MDO implementation in industry. Another barrier is the unavailability and lack of MDO experts in industry; those who understand the MDO process and know the implementation tasks involved.

In an endeavor to address the mentioned implementation challenges, a novel collaborative multidisciplinary design optimization (CMDO) framework is defined in order to be applied in the conceptual design phase. CMDO provides a platform where many engineers team up to increase the likelihood of more accurate decisions being taken early on. The structured way to define the engineering responsibilities and tasks involved in MDO helps to facilitate the implementation process.

It will be further elaborated that educating active engineers with MDO knowledge is an expensive and time-consuming process for industries. Therefore, a guideline for CMDO implementation in conceptual design is proposed in this thesis that can be easily followed by design engineers with limited prior knowledge in MDO. The performance of the framework is evaluated in a number of case studies, including applications such as aircraft design and the design of a tidal water power plant, and by engineers in industry and student groups in academia. 
Have patience, all things are difficult before become easy.

Saadi Shirazi (1203-1291) 


\section{Acknowledgements}

There are many individuals and organizations that I would like to truly thank for their support and valuable advice during the course of this work.

Firstly, I would like to express my special thanks to my supervisor Professor Johan Ölvander, Head of the Division of Machine Design, who gave me the opportunity to work in the research group. Your commitment, motivation, enthusiasm and patience as a supervisor have always been appreciated. Your guidance helped me throughout the research and the writing of this thesis.

Special thanks go to my co-supervisor and friend Dr. Mehdi Tarkian for stimulating discussions, sharing his experience, and contributing to this work. Your support and care helped me overcome delays and stay focused on the work.

I am grateful to Prof. Hampus Gavel, my former industrial co-supervisor, for the valuable discussions that have led me to better understanding and enriched my ideas. He has always been an indispensable source of inspiration and trust. I would further like to thank Prof. Petter Krus, my former co-advisor, for all fruitful discussions that we have had.

Alongside my supervisors, my sincere thanks also go to all of my wonderful colleagues at the Division of Machine Design and Fluid and Mechatronic Systems. Thank you for offering me a wonderful workplace with an enjoyable environment.

I would also like to acknowledge the support given by our industrial partners, SAAB Aeronautic, Mineso AB and ABB Corporate Research, and especially Prof. Xiaolong Feng. I would also like to thank the Swedish Defense Materiel Administration (FMV) and Sweden's innovation agency (VINNOVA) for financially supporting this work.

Last but not least, I would like to express my gratitude to my family for always believing in me. In particular, my parents for their sacrifices and their encouragement that have made everything I have done possible. Special thanks go to my wonderful wife Aida for always supporting me unconditionally, and my son Ryan for bringing unexplainable taste to my life. Aida, you were the one who maintained the foundation of our family and made personal sacrifices to bring happiness to our life, and I understand it has at times been very difficult for you, especially in recent years. This work is dedicated to you.

Edris Safavi

Linköping, October, 2016 
Your task is not to seek for love, but merely to seek and find all the barrier within yourself that you have built against it. Jalal ad-Din Muhammad Balkhi (Rumi), (1207-1273)

viii 


\section{Appended Papers}

The following papers are appended and will be referred to by their Roman numerals.

[I] Safavi E., Gopinath V., Ölvander J., and Gavel H., "A Collaborative Tool for Conceptual Aircraft Systems Design", AIAA Modelling and Simulation Technologies, Minneapolis, Minnesota, USA, 2012.

[II] Safavi E., Tarkian M., Gavel H., and Ölvander J., "Collaborative Multidisciplinary Design Optimization: A Framework Applied on Aircraft Conceptual System Design", Journal of Concurrent Engineering, 23: 236-249, doi:10.1177/1063293X15587020, first published on June 3, 2015.

[III] Safavi E., Tarkian M., Ölvander J., Najafabadi H. N., and Chaitanya M. V., "Implementation of Collaborative Multidisciplinary Design Optimization for Conceptual Design of a Complex Engineering Product", Journal of Concurrent Engineering doi:10.1177/1063293X16661224 first published on August 1, 2016.

[IV] Safavi E., Tarkian M., and Ölvander J., "A Guideline to Facilitate the Implementation of Collaborative $M D O$ in Conceptual Design of Complex Products", Submitted for journal publication, 2016.

[V] Chaitanya M. V., Najafabadi H. N., Safavi E., Ölvander J., Krus P., and Karlsson M., "A Comprehensive Computational MDO Approach for A Tidal Power Plant Turbine", Submitted for journal publication, 2016.

[VI] Safavi E., Tarkian M., Ölvander J., "Rapid Concept Realization for Conceptual Design of Modular Industrial Robots", NordDesign2010, Göteborg, Sweden, 2010.

\section{Note:}

The appended papers are printed in their originally published style except for some minor reformatting to fit to the layout of the dissertation. 


\section{Author's Contributions}

In papers [I] to [III], Safavi conducted the research, created the simulation models, developed the MDO framework, and prepared the manuscript. In paper [IV], Safavi collected the material, developed the method, and acted as lead author in preparing the manuscript. In paper $[\mathrm{V}]$, Safavi developed the multidisciplinary design optimization framework, created the dynamic simulation models, ran the optimization, and interpreted the results. Safavi also contributed extensively to prepare the manuscript. Safavi is the main author for paper [VI]. He is responsible for improving the CAD models, developing dynamic models, constructing the physical demonstrator, and running the experiment and evaluating the results. 
The following papers are not included in the thesis but constitute an important part of the background.

[VII] Safavi E., Gopinath V., Ölvander J., Gavel H., "Conceptual Optimization of Aircraft Actuator Systems", Recent Advances in Aerospace Actuation Systems and Components, Toulouse, France, 2012.

[VIII] Safavi E., Chaitanya M. V. R., Ölvander J., Krus P., "Multidisciplinary optimization of Aircraft Actuation System for Conceptual Analysis", AIAA 51st Aerospace Sciences Meeting, Grapevine, Texas, 2013.

[IX] Safavi E., Namakian M., Sirén T., Magnéli R., and Ölvander J., "Design and Evaluation of Airborne Wind Turbine Utilizing Physical Prototype", International Congress on Energy Efficiency and Energy Related Materials (ENEFM2013): Proceedings, Springer, pp 57-64, 2013. 
We can't solve problems by using the same kind of thinking we used when we created them. Albert Einstein (1879-1955) 


\section{Abbreviations}

$\begin{array}{ll}\text { AAO } & \text { All at Once } \\ \text { AK } & \text { Anisotropic Kriging } \\ \text { CAD } & \text { Computer Aided Design } \\ \text { CAE } & \text { Computer Aided Engineering } \\ \text { CAVE } & \text { Collaborative Aircraft Vehicle Engineering } \\ \text { CCD } & \text { Collaborative Conceptual Design } \\ \text { CD } & \text { Conceptual Design } \\ \text { CDO } & \text { Conceptual Design Optimization } \\ \text { CE } & \text { Conceptual Engineer } \\ \text { CFD } & \text { Computational Fluid Dynamics } \\ \text { CMD } & \text { Conceptual Multidisciplinary Design } \\ \text { CMDO } & \text { Collaborative Multidisciplinary Design Optimization } \\ \text { CO } & \text { Constraint } \\ \text { DC } & \text { Data Centric } \\ \text { DE } & \text { Domain Expert } \\ \text { DFA } & \text { Disciplinary Feasible Analysis } \\ \text { DOE } & \text { Design of Experiment } \\ \text { DS } & \text { Descriptive Study } \\ \text { FEM } & \text { Finite Elements Modeling } \\ \text { FMI } & \text { Functional Mockup Interface } \\ \text { GA } & \text { Genetic Algorithm } \\ \text { HFM } & \text { High Fidelity Model } \\ \text { HSD } & \text { Hierarchical System Decomposition } \\ \text { IC } & \text { Interface Centric } \\ \text { IDF } & \text { Individual Disciplinary Feasible } \\ \text { IE } & \text { Interface Expert } \\ \text { LFM } & \text { Low Fidelity Model } \\ \text { MDF } & \text { Multi-Disciplinary Feasible } \\ \text { MDO } & \text { Multidisciplinary Design Optimization } \\ \text { MFA } & \text { Multidisciplinary Feasible Analysis } \\ \text { MOGA } & \text { Multi-Objective Genetic Algorithm } \\ \text { NHSD } & \text { Non-Hierarchical System Decomposition } \\ \text { OB } & \text { Objective } \\ \text { OE } & \text { Optimization Expert } \\ \text { PS } & \text { Prescriptive Study } \\ \text { ULH } & \text { Uniform Latin Hypercube } \\ & \end{array}$


Yesterday I was clever, so I wanted to change the world. Today I am wise, so I am changing myself. Jalal ad-Din Muhammad Balkhi (Rumi) , (120\%-1273) 


\section{Contents}

1 Introduction $\quad 1$

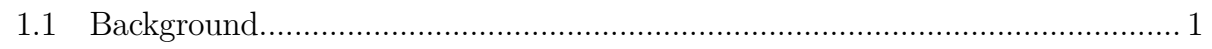

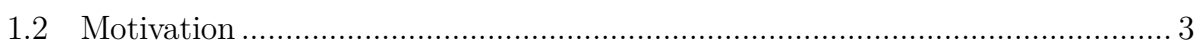

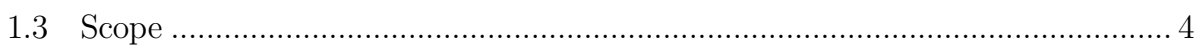

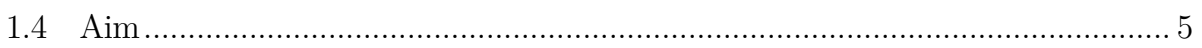

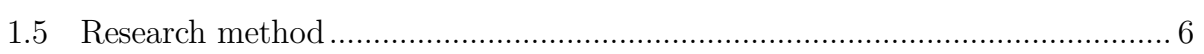

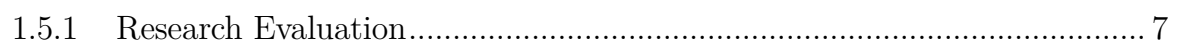

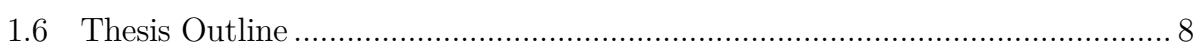

2 Theoretical Background $\quad 9$

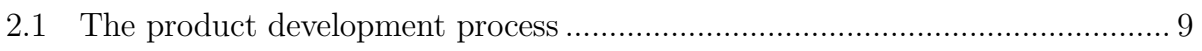

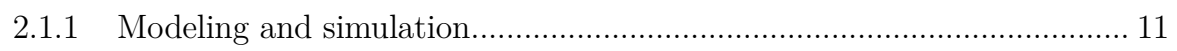

2.1.2 Computer Aided Engineering (CAE) ...................................................... 11

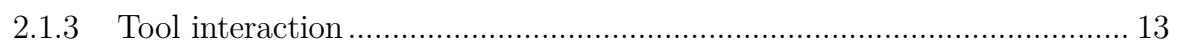

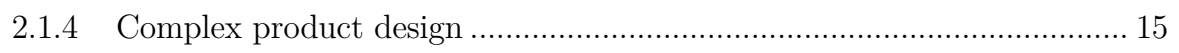

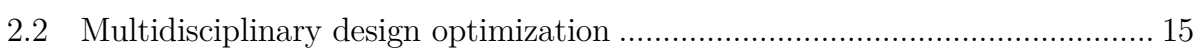

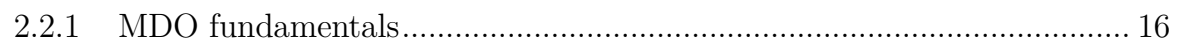

2.2.2 MDO in conceptual design.............................................................. 17

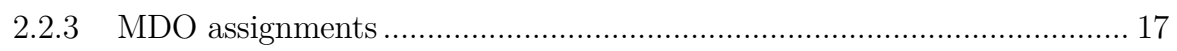

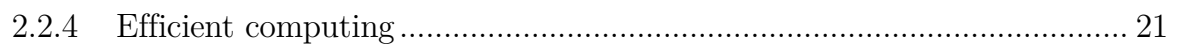

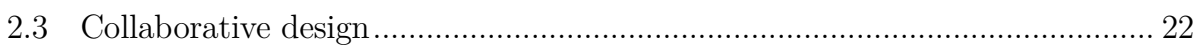

3 Contributions $\quad \mathbf{2 5}$

3.1 Collaborative multidisciplinary design optimization (CMDO) ...................... 26

3.1.1 Roles and responsibilities in a CMDO framework .............................. 26

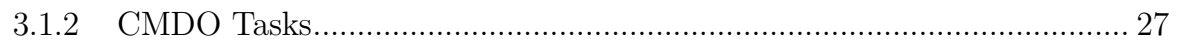

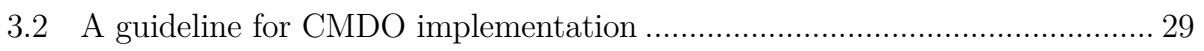

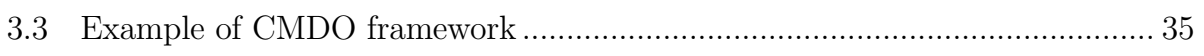

4 Summary of the appended papers $\quad 39$

4.1 Paper I: A Collaborative Tool for Conceptual Aircraft Systems Design ........ 39

4.2 Paper II: Collaborative Multidisciplinary Design Optimization: A Framework Applied on Aircraft Conceptual System Design........................................................ 41 
4.3 Paper III: Implementation of Collaborative MDO for Conceptual Design of a Complex Engineering Product ............................................................................... 42

4.4 Paper IV: A Guideline to Facilitate the Implementation of Collaborative MDO in Conceptual Design of Complex Products ............................................... 43

4.5 Paper V: A Comprehensive Computational MDO Approach for a Tidal Power

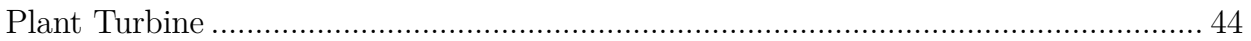

4.6 Paper VI: Rapid Concept Realization for Conceptual Design of Modular

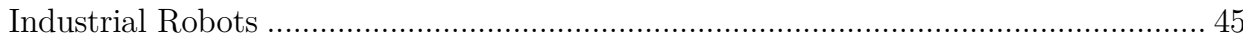

$\begin{array}{ll}5 \text { Discussion } & \mathbf{4 7}\end{array}$

5.1 Collaborative Aircraft Vehicle Engineering (CAVE) and the idea of CMDO 47

5.2 CMDO and challenges to its implementation .............................................. 49

5.3 Generalization and industrial use of CMDO ............................................... 50

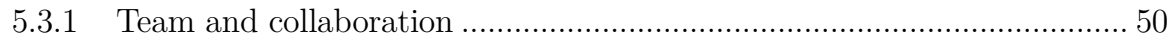

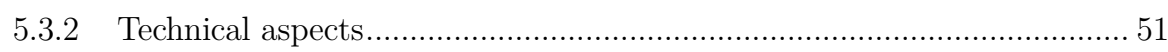

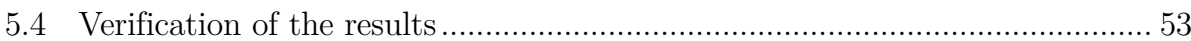

6 Conclusions $\quad 55$

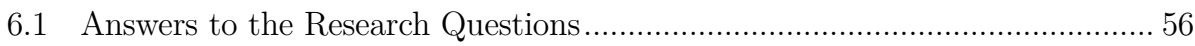

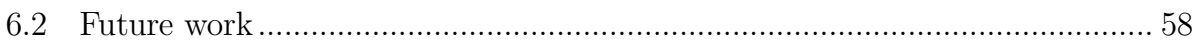

$\begin{array}{ll}\text { References } & 59\end{array}$ 


\section{1 \\ Introduction}

$\mathrm{D}$

ESIGN of complex engineering products such as aircraft, cars, robots, or trains is a challenging multidisciplinary task that engages a range of human, computing as well as manufacturing resources. Competition in the global market forces manufacturers to develop optimized products faster and more cheaply that ensure customer satisfaction. It goes without saying that this task is best achieved by employing efficient processes to explore the design space and generate innovative products.

Multidisciplinary design optimization (MDO) has proven to be a promising technique to efficiently manage complex designs with many interacting disciplines. MDO has been a hot research topic for many years and is applied in industry today. MDO can be applied in various stages of design, for broader or more specific focus, for one or several departments.

This thesis will explore and investigate the prospect of applying MDO in the conceptual design (CD) phase. MDO will serve as an enabler to apply higher fidelity models (HFMs) earlier and thus raise the level of concept information. The thesis will hence suggest guidelines to overcome technical and organizational barriers to implementing MDO and HFMs in practical engineering settings.

\subsection{Background}

An engineering design process starts with conceptual design, where various design concepts are selected to be further studied and optimized with respect to a set of initial requirements (Brandt et al., 1997). The selected concepts that fulfill the requirements are further analyzed in later design phases, e.g. preliminary or detail design phases (Ulrich and Eppinger, 2016). The information gathered during the conceptual phase is therefore vital to create a wider product knowledge foundation. Henceforth, gathering more information 
in the conceptual phase is valuable and helps decisions to be made with less cost overruns. This is even more critical for complex and unconventional products with limited prior empirical and analytical information.

Typically, in most product development processes, the more knowledge gained the less freedom is left to practically apply the knowledge in the concept development, see figure 1-1. This is mainly due to increasing fidelity of the design models and the increasing complexity of the design process, which includes various teams and departments. More accurate and fundamental design decisions in the conceptual phase may thus lead to an overall cheaper and faster product development. However, in order to do so successfully, the fidelity of the conceptual analysis should be increased.

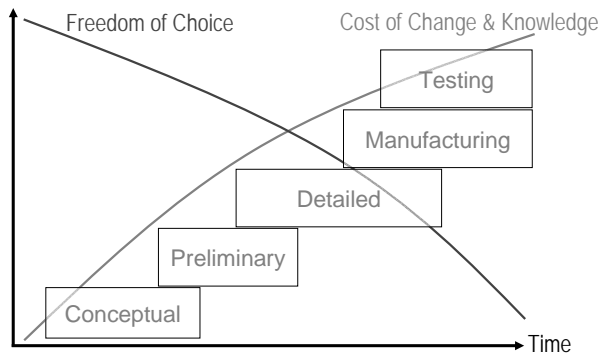

Figure 1-1: The Information and freedom paradox in a design and manufacturing process, adapted from (Jenkinson et al., 1999; Mavris et al., 2000).

Conceptual engineers normally deal with lower fidelity models (LFMs) to rapidly configure the product concepts and evaluate them holistically. Although LFMs are faster and easier to develop, they provide limited detail information regarding technology, features, and functionality of the concepts under evaluation (Wang et al., 2002). Detail information is normally produced in preliminary and detail design stages when design questions are more specific and higher fidelity tools are accessible. HFMs in CD could effectively raise the information level in early design phases.

On the other hand, HFMs also increase the complexity of the design process. This could even make the decision-making process more time-consuming. Consequently, the lead- time would become worse. This can be overcome by applying MDO, which is known to be an effective tool to search through complex design spaces.

In order to be able to apply HFMs and MDO in the conceptual design phase, a collaborative effort is needed at the company. Collaborative design is described as a process where a product is designed through the joint efforts of various domain experts (Wang et al., 2002). Collaborative design in this thesis is used to facilitate the work done in interaction between the domain experts and conceptual engineers to effectively integrate 
high fidelity design tools in CD. For more information regarding collaborative design see chapter 2.3 .

Collaborative multidisciplinary design optimization is therefore presented in this thesis as a framework that:

- Includes high fidelity models

- Enables the implementation of MDO

- Is executed in a collaborative process combining conceptual engineers with domain experts.

\subsection{Motivation}

Today's competition among industries is about developing more innovative and optimized products. This can be achieved by gathering more information about the product prior to manufacturing and reflect it efficiently to improve the design. A popular way to collect more information is to increase the fidelity of the design models early in the process. As mentioned earlier, MDO has been shown to be an effective tool to assist the engineers to explore the design space and generate more knowledge about the concept under evaluation. However, the use of MDO in industry is still limited as Agte et al. (2009) reported from European-US Multidisciplinary Optimization Colloquium in 2006 where almost seventy professionals from academia, industry and government gathered together. The authors mentioned that MDO in industry is hampered due to barriers of a technical, organizational, cultural, and educational nature. A couple of years later, 48 researchers from both academia and industry who are active in MDO research identified and categorized the challenges that face MDO implementation into 5 distinct topics, which Simpson and Martins (2011) reported as:

1. Modeling and design space

2. Metrics, objectives, and requirements

3. Coupling of complex engineered systems

4. Dealing with uncertainty

5. People and workflow

The challenges involving people and workflow together with modeling and coupling strategies are the most common challenges that other researchers have also recently mentioned as future work within MDO (Hoogreef and La Rocca, 2015; SobieszczanskiSobieski et al., 2015). Therefore, among the interesting topics mentioned above, this thesis focuses on challenges of modeling and coupling and on how to efficiently involve different engineers in an MDO framework.

The studies above also highlight the fact that industry is suffering from a lack of engineers who understand MDO and methods for implementing it. 
In summary, the objective of this research is to develop a method that assists engineering teams in implementing MDO and HFMs in an early design phase - conceptual design with fewer challenges.

\subsection{Scope}

Classical MDO is used in preliminary and detail design phases (Dieter et al., 2003; Sobieszczanski-Sobieski et al., 2015). However, MDO in this thesis is considered to help the conceptual engineers to expand the product knowledge foundation. As figure 1-2 illustrates, engineering departments at manufacturing companies are divided into different sections named conceptual, preliminary, detail, prototyping, etc. (Mavris et al., 2000). Regularly, the human resources of engineering departments are unequally speared into various departments. For instance, the ratio between the number of conceptual engineers to domain experts in a manufacturing company of complex and hi-tech products is perhaps one to tens or hundreds. This disproportionately puts a heavy workload and responsibility on conceptual engineers considering, single errors can waste thousands of man-hours' work or even result in project failure.

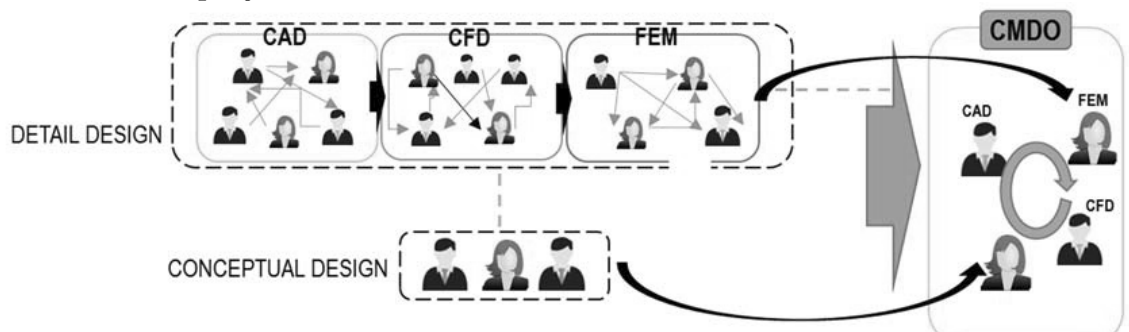

Figure 1-2: Schematic of engineering departments at manufacturing company and their contribution to CMDO framework development.

Collaborative multidisciplinary design optimization (CMDO) is proposed in this thesis to facilitate the mentioned collaboration and integration. As illustrated in figure 1-2, CMDO provides a platform to facilitate the integration of the domain expert's knowledge into conceptual design and increase the product knowledge.

Figure 1-3 illustrates how CMDO relates to other scientific disciplines in an engineering design process. As mentioned earlier, CMDO in this thesis aims to improve the efficiency of conceptual design as one of the most important parts of the design process by bringing HFMs into CD (Wang et al., 2002; Lilienthal, 2003). This thesis deals with complex and multidisciplinary products and multidisciplinary design is therefore used to develop the product considering various perspectives. Since the design space is extended significantly when HFMs are used, multidisciplinary design is ineffectual without integration with design optimization to search the extended design space for optimal concepts. This is where the area of MDO is situated (Giesing et al., 1998). In fact, it is here proposed that 
CMDO be used as an MDO enabler. Collaborative design is used as a facilitator of such a process (Kvan, 2000; Peng, 1994). Hence, the research interests in the thesis lie at the intersection of where multidisciplinary design optimization applies collaboratively in conceptual design, here called CMDO. A more detailed discussion of the adjacent research areas will be given in chapter 2 .

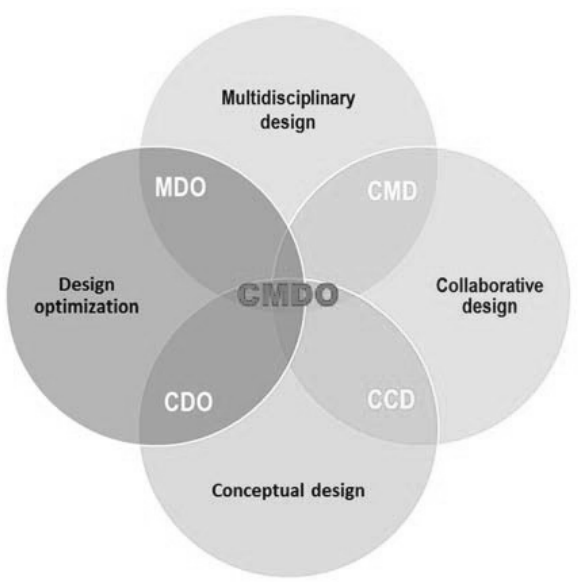

Figure 1-3: How CMDO relates to other research topics.

\subsection{Aim}

This thesis deals with the collaborative process for practical and engineering enabled implementation of MDO, which leads to:

- Increase of knowledge in early design phases by using higher fidelity models

- Easier implementation of MDO within engineering teams

- Reduction of uncertainty during conceptual design

- Automated design space exploration in conceptual design

The principal research questions (RQ) can be formulated as:

RQ1: How can the usage of high fidelity models be supported in the conceptual phase?

RQ2: What are the important technical and non-technical barriers to implement MDO in an engineering team and how can they be managed?

RQ3: How can efficient implementation of an MDO framework in an engineering team be supported? 


\subsection{Research method}

Scientists have always tried to find the perfect classification of science. This has been a continual dilemma since 415 B.C. when Plato began to define and classify science into geometry, math, and art (Zeyl, 2000). Even though the classifications are dynamic and ever changing, it is still crucial to classify the created scientific output.

Scientific method is defined by the Oxford English Dictionary (2016), as " a method or procedure that has characterized natural science since the 17th century, consisting in systematic observation, measurement, and experiment, and the formulation, testing, and modification of hypotheses." To better formulate the scientific method, this research follows the design research methodology (DRM) suggested by Blessing and Chakrabarti (2009), see figure 1-4. The process consists of four different steps:

- Goal: where a metric that measures the success of the study is provided.

- Descriptive study 1 (DS1): where the current problem is studied using available methods and materials to identify the factors that influence the formulated goal.

- Prescriptive study 1 (PS1): where methods and tools to solve the identified problems in DS1 are developed using experience and assumptions.

- Descriptive study 2 (DS2): where the effect of developed tools or methods on the influencing factors are evaluated and verified. The result is analyzed and employed to improve the developed method (feedback 1) or to do success evaluations by a comparison with the influencing factors resulting from DS1 (feedback 2). Application analysis, collection of empirical data and observations are used as basic methods to perform DS2.

The steps above can be iterated to achieve proper accuracy and validity of the developed methods and tools.

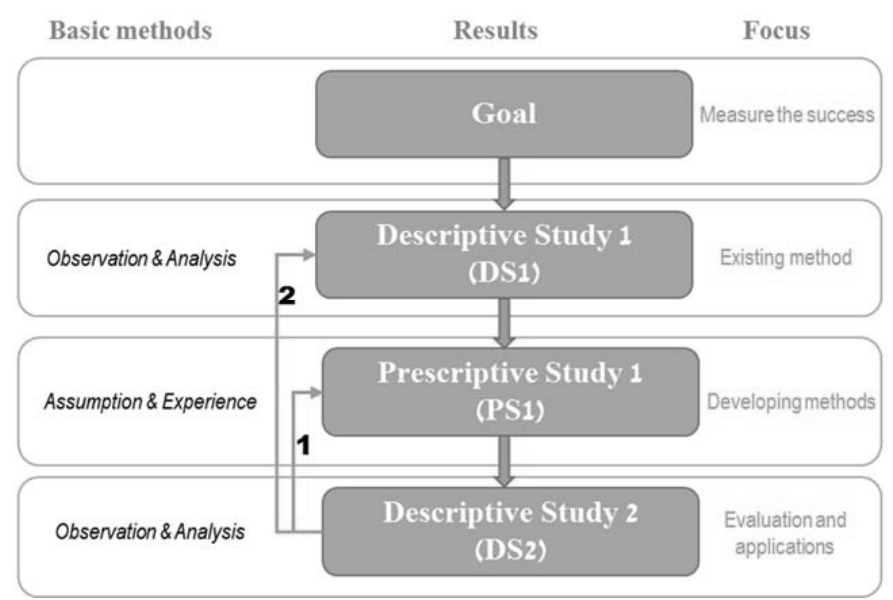

Figure 1-4: Design research methodology framework (Blessing and Chakrabarti, 2009). 
The aforementioned research method is used throughout this study with only minor deviations. The connection of the research method to the different studies reported in the appended papers is illustrated in table 1-1. The table explains how the appended papers and the implemented research method are related to each other and to the research questions. The first column on the left side of the table describes different steps of the employed research method where the goal of the study of each paper is defined. Explicitly, the contribution of each paper to answer the research question is described in the second row. The analysis of the goal using existing method is evaluated in the DS1 row. The existing methods are those proposed in literature or previous papers of the author. Then the developed method in each paper is presented in the method's row (PS1). Finally, the developed method is analyzed and evaluated based on predefined goal using various application studies. For example, creating more knowledge in conceptual design is defined as goal for paper 1 . The state of the art and some previous case study is evaluated with respect to the goal and to highlight the research gap. An exploratory case study is used to propose the method to fill the gap, and the result is evaluated in a case study from aircraft conceptual system design.

Table 1-1: Overview of the links between the appended papers, research methods and research questions.

\begin{tabular}{|c|c|c|c|c|c|c|}
\hline & Paper I & Paper II & Paper III & Paper VI & Paper V & Paper VI \\
\hline Goal & $\begin{array}{l}\text { Increase } \\
\text { product } \\
\text { knowledge } \\
\text { foundation } \\
\text { in CD }\end{array}$ & $\begin{array}{l}\text { Evaluation } \\
\text { of CAVE } \\
\text { within an } \\
\text { MDO } \\
\text { framework }\end{array}$ & $\begin{array}{c}\text { Evaluation } \\
\text { and } \\
\text { improvement } \\
\text { of CMDO } \\
\text { framework }\end{array}$ & $\begin{array}{l}\text { Development of } \\
\text { an } \\
\text { implementation } \\
\text { guideline for } \\
\text { CMDO }\end{array}$ & $\begin{array}{l}\text { Effective use of } \\
\text { HFMs in complex } \\
\text { MDO setting }\end{array}$ & $\begin{array}{l}\text { Performance } \\
\text { and } \\
\text { validation of } \\
\text { HFM:s in } \\
\text { CD } \\
\end{array}$ \\
\hline Coverage & RQ1 & RQ1 \& 3 & RQ2 \& 3 & RQ $2 \& 3$ & RQ1 & RQ1 \\
\hline $\begin{array}{c}\text { Existing } \\
\text { method } \\
\text { (DS1) }\end{array}$ & $\begin{array}{c}\text { Literature \& } \\
\text { Previous } \\
\text { Case studies }\end{array}$ & & Paper II & $\begin{array}{c}\text { Literature \& } \\
\text { Paper II, III, IV }\end{array}$ & $\begin{array}{c}\text { Literature (non- } \\
\text { relational } \\
\text { parameterization) }\end{array}$ & $\begin{array}{c}\text { Literature } \\
\text { (Analytical } \\
\text { method) }\end{array}$ \\
\hline $\begin{array}{c}\text { Developing } \\
\text { Methods } \\
\text { (PS1) }\end{array}$ & CAVE & $\begin{array}{c}\text { CMDO } \\
\text { (integration } \\
\text { of CAVE } \\
\text { and MDO) }\end{array}$ & $\begin{array}{l}\text { Improved } \\
\text { CMDO }\end{array}$ & $\begin{array}{c}\text { CMDO } \\
\text { implementations' } \\
\text { guideline, }\end{array}$ & $\begin{array}{c}\text { Relational } \\
\text { parameterization }\end{array}$ & $\begin{array}{c}\text { Rapid } \\
\text { concept } \\
\text { realization }\end{array}$ \\
\hline $\begin{array}{c}\text { Evaluations } \\
\text { and } \\
\text { application } \\
\text { analysis } \\
\text { (DS2) }\end{array}$ & $\begin{array}{c}\text { Exploratory } \\
\text { case study, } \\
\text { Aircraft } \\
\text { system } \\
\text { design }\end{array}$ & $\begin{array}{c}\text { Exploratory } \\
\text { case study, } \\
\text { Aircraft } \\
\text { system } \\
\text { design }\end{array}$ & $\begin{array}{c}\text { Exploratory } \\
\text { case study } \\
\text {,Tidal water } \\
\text { plant }\end{array}$ & $\begin{array}{l}\text { Exploratory case } \\
\text { study, Multiple } \\
\text { applications }\end{array}$ & $\begin{array}{c}\text { Exploratory case } \\
\text { study, Tidal } \\
\text { power plant }\end{array}$ & $\begin{array}{c}\text { Exploratory } \\
\text { case study, } \\
\text { Industrial } \\
\text { robot }\end{array}$ \\
\hline
\end{tabular}

\subsubsection{Research Evaluation}

The difference between the research work and engineering work is among others that research should provide new, reliable and reusable knowledge for others (Cross, 2007). The results of the design research, which could be in different forms such as methodology, 
processes, design tool as well as models therefore need to be validated. However, validation of design research is hard (if not impossible) using classical experiments and observations due to the high number of control factors and the unpredictability of the results of the design process in each iteration. Hence, Buur (1990) suggests two approaches to evaluate the quality of design research: logical verification and verification by acceptance. Evaluation and the reliability of the results are verified by logical verification when the different factors such as methodological thoroughness and theoretical consistency within the research as well as results from external research are considered. Verification by acceptance, however, considers how new scientific results are accepted by other researchers and experienced engineers within the field. A discussion of validity and verification of the methods developed in this thesis can be found in Section. 5.4.

\subsection{Thesis Outline}

The dissertation is constructed as a compilation thesis and includes an introduction and six appended papers. The scientific basis of the appended papers is thoroughly explained in the introductory section, which is also intended to provide a summary of the theory, methods, and results presented in the appended papers.

The introductory section consists of 5 chapters:

Chapter 1 introduces the background to the work and the research questions and research methods.

Chapter 2 extends the frame of reference of the involved domains in this research.

Chapter 3 presents the contributions and describes the developed methods. The methods are utilized in various industrial applications for thorough evaluation and validation.

Chapter 4 contains a summary of the contribution of each appended paper in the creation and implementation of the proposed methods.

Chapter 5 Discusses previous work and the contributions of the thesis, limitation and generalization of the proposed methods and finally verification of the results.

Chapter 6 concludes the thesis with answers to the research questions as well as some suggestions for future work. 


\section{2 \\ Theoretical Background}

7 HIS chapter consists of a review of existing and relevant literature, highlighting the gaps that the present work intends to fill. The important theoretical concepts are briefly introduced as a foundation for the contributions that are presented in chapter 3. The intention of the work as presented in the first chapter is to bring higher fidelity models into conceptual design. The definition of HFMs and their position in the design process therefore need to be clarified (see Section 2.1). The complexity of dealing with such models and method to manage it is another topic which is discussed in Section 2.2. Finally, development and integration of HFMs in the design process require extensive collaboration among engineers as described in Section 2.3.

\subsection{The product development process}

The product development process defined by Pahl and Beitz (1996) starts from planning and task-setting. This is then followed by design activities, prototyping and manufacturing as well as testing and modification, etc. This thesis is focused on the design activity parts that can schematically be divided into three phases: conceptual, preliminary and detail design (Pahl et al., 2007).

- Conceptual design: where various concepts are generated and evaluated based on the predefined problem specification. The best concepts are selected for further investigation.

- Preliminary design: where the selected concepts are further evaluated and given an embodiment shape. 
- Detail design: where the preliminary concept is detailed, analyzed and optimized with respect to various requirements. The concept is prepared for prototyping.

Conceptual design has a direct impact on both the preliminary and the detail design phases (Ullman, 2003). Every minor error in this phase may result in high design costs and time overruns. As illustrated in figure 2-1, more than $70 \%$ of the total cost of a product's development is locked and determined as early as the conceptual phase. However, the actual design cost is spent later on (Lilienthal, 2003). This highlights the importance of the CD phase in the design process. New methods to create more knowledge in CD will thus benefit the whole product design process.

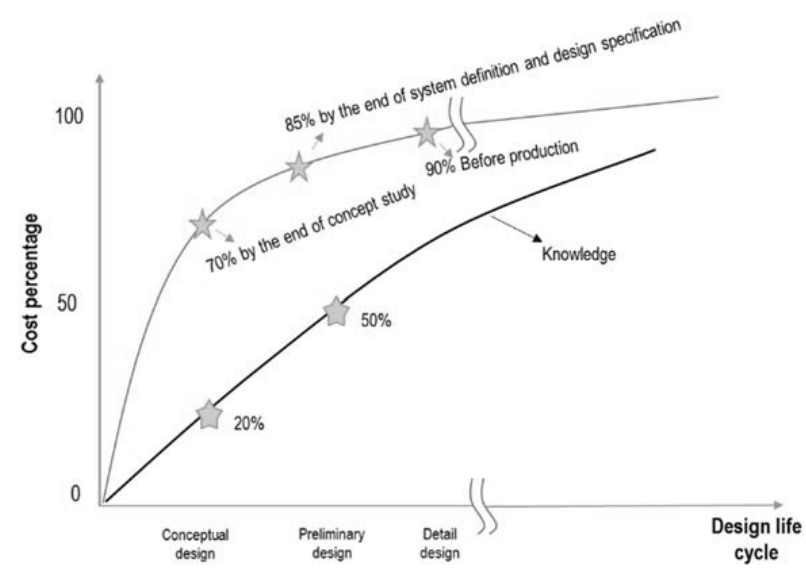

Figure 2-1: Product cost locked associated with early stages of the design process (adapted from Lilienthal, 2003).

Few design tools are available in the conceptual phase and these are immature and undeveloped compared to the tools in the detail phase, see figure 2-2. This provides a great opportunity for developing new tools or reusing the existing tools, e.g. bringing the high fidelity tools used in detail design like CAD and CAE into conceptual design (Wang et al., 2002).

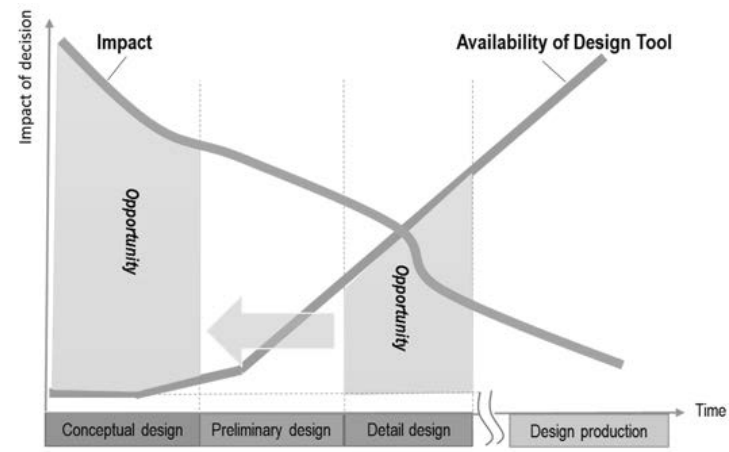

Figure 2-2: Impact of design phases and availability of design support tools in different design stages (Adapted from Wang et al., 2002). 


\subsubsection{Modeling and simulation}

As presented by Raynould (1996), "a model is a representation of a system that replicates part of its form, fit, function or a mix of the three, in order to predict how the system might perform or survive under various conditions". A model thus plays a vital role in measuring the outcomes when real-life experiments are impossible or impractical.

A model represents a system and a simulation is the execution of the model over a specific period of time; simulation is thus about performing a physical or a virtual experiment on the model. A behaviour prediction of the product can therefore be achieved by performing simulation on the model in various conditions. By altering the fidelity level of the simulation model, the precision of the results can be modified. Simulation models are widely used in industry because of proven benefits in comparison to physical tests such as cheaper, faster, safer, and easier to generate and analyse the results. Simulation models therefore play an essential role in the design process of today.

A conceptual model as defined by Robinson (2008) is "a non-software specific description of the computer simulation model (that will be, is or has been developed), describing the objectives, inputs, outputs, content, assumptions and simplifications of the model". Hence, the conceptual models are the abstractions of the detail simulation models.

\subsubsection{Computer Aided Engineering (CAE)}

The significant benefit of computerization of design to increase accuracy and reduce time is no more a question. Computer aided engineering (CAE) is defined as the use of computer software to help engineers better analyse the task (Daintith and Wright, 2008). CAE contains many disciplines, e.g. CAD, CFD, FEM, and Dynamic Simulation. Each discipline is supported by a number of different software tools. This thesis has dealt with some of the disciplines and associated tools that are briefly presented below:

\section{Computer aided design (CAD)}

Geometric models have great potential to be used in CD to provide more information about the systems and their components (Tarkian, 2012; Amadori 2012). Various design properties can be better estimated utilizing geometric modeling. Detailed geometric information regarding the final concept is very limited in conceptual design. Computer Aided Design (CAD) tools are therefore traditionally used in later stages of the design (Ledermann et al., 2005). However, to bring CAD into conceptual design, simplifications need to be applied. On the other hand, the simplified models need to be refined and changed repeatedly in order to define accurate models and evaluate different concepts.

Parametric modeling is defined to make more flexible models (Berard et al., 2008). The term parametric modeling denotes the use of parameters to control the CAD models. Some features such as the dimensions and shape of the design can be controlled using 
parameters. The advantage of parametrization fits the requirements of conceptual design, which is to rapidly generate and evaluate concepts. Another advantage of parametric design is in optimization where the design parameters are defined as variables to be changed rapidly by an optimization algorithm.

In general, geometric modifications made on a CAD model will either alter the shape of the elements (morphology) or the number of elements (topology), (Amadori, 2012; Tarkian, 2012). Parameterization can thus be defined for both morphology and topology of the elements. Topological parameterization is accomplished by defining templates and context manuals, see figure 2-3. For more information regarding topological parameterization, see (Tarkian 2012). CATIA (Dassault system (1), 2016), Solidwork (Dassault system (3), 2016) and CREO (ProEngineer, 2016) are some of the commercial software tools to support CAD. They are widely used in industry. CATIA v5 is used in this thesis for geometric design.
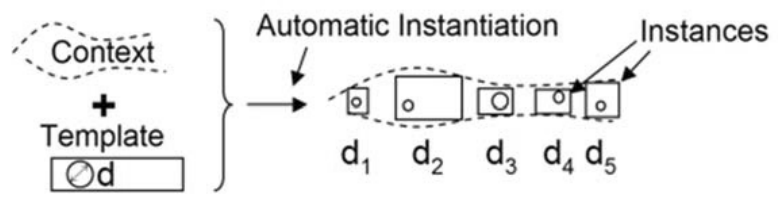

Figure 2-3: Topological instantiation by defining a template and a context, adapted from (Tarkian, 2009).

\section{Computational Fluid Dynamics (CFD)}

Mathematical relationships and physics (based on the Navier-Stokes equations) are used in Computational Fluid Dynamics to simulate the dynamic behavior of a fluid with respect to different conditions (Ferziger and Peric, 2002). These equations describe how the velocity, pressure, temperature, and density of a moving fluid are related. In general, analyzing a fluid flow problem using commercial CFD tools may include pre-processor, solver, and post-processor elements. The first element includes creation of the geometry and mesh generation. The selection of physics and fluid properties, specification of boundary conditions, and solver set-up (such as solution control, monitoring the solution, convergence control, etc.) form the second element. Finally, within the post-processing, the results are interpreted by means of different types of plotting tools, etc. Each of these elements contains a number of components for which the way they are treated will contribute to the validity, accuracy and computational cost of the CFD analysis. Nowadays, there is a strong connection between CAD and CFD tools to exchange geometric models. Similar to CAD, CFD also benefits significantly from parameterization (Chawner et al., 2016; Vasilopoulos et al., 2016; DesJardin et al., 2016). There are many commercial CFD tools available, for example ANSYS Fluent (Fluent, 2016), Star-CCM+ (CD-adapco, 2016), and IESVE Mcroflo (Microflo, 2016). ANSYS Fluent is used in in this thesis in papers III, IV and V for CFD analysis. 


\section{Finite Element Method (FEM)}

FEM uses numerical techniques to solve the boundary value problems in for example solid mechanics (Reddy, 2005). FEM is extensively used in the structural analysis discipline and helps to visualize the stiffness and strength of the products. Nowadays, there are several FE commercial toolkits for specific domains such as thermal, electromagnetic and fluid analysis.

Similar to CFD analysis, the problem in FEM is subdivided into smaller and simpler elements called mesh to be solved more easily. The equations applied on simple elements are then assembled to create the larger system of equations representing the entire problem. Similar to CFD, FEM also consists of pre-processing, solver and post-processing parts (Dugan, 2016). Abaques (Simulia, 2016), ANSYS Mechanical (ANSYS Mechanical, 2016) are some of the examples of FE software tools that are widely used in industry. ANSYS Mechanical release 15 is used in the application study presented in the appended papers.

\section{Dynamic system simulation}

Dynamic models normally use differential equations to calculate and present the behavior of a system over time. A variety of commercial tools are available which are specialized to different domains, e.g. Adams for mechanical systems (MSC software, 2016) or Dymola for modeling complex and multi-domain systems (Dassault System (2), 2016) and MATLAB Simulink for multi-domain simulation and model-based design (Mathworks, 2016).

In this study, Dymola is used to create the dynamic models and run the simulation. Dymola is based on the Modelica language. Modelica is an object-oriented language used to model complex physical systems (Modelica Association, 1999; Fritzson, 2004).

Nowadays, most of the commercial tools are supported by extensive model libraries for different engineering domains. Different elements of a system are modeled and stored in the model library. The engineers can easily use and integrate these models to construct various system models.

\subsubsection{Tool interaction}

Model development for multidisciplinary analysis requires specific considerations. This is mainly due to the new requirements demanded by coupling the models. The flexibility and robustness of the models are very important factors in smoothly interacting in a multidisciplinary design process (Amadori, 2012). There are different methods to help the designer to make the design more flexible, for example parametric and modular design.

The main purpose of parametrization according to Sunnersjö et al. (2006) is "to allow reuse of existing design solutions with adaptations to new specifications". The expression of parameters in multidisciplinary design clarifies the relationship between design intent 
and design response (Woodbury, 2010). According to Woodburry, the parametric modeling method can be divided into two categories as follows.

Propagation-based systems, where data flow helps the series of changes start from one parameter to the others. In fact, it initiates from knowns and computes the unknowns using history-based method where a record of how the model was built is stored. When the changes are applied on parameters and regeneration is requested for the part, the same operations from the history are repeated to create the new part.

Constraint systems, where a set of continuous and discrete constraints are solved. The constraints can be used to create relationships between parameters and ensure that the new model is always kept in the design space and fulfills the constraints. This is done by defining relationship between the parameters if one or several parameters use the same value, or depend on the values of several others (Woodbury, 2006).

Apart from interdisciplinary interaction (inside each discipline), which may be controlled by parametric modeling, the multidisciplinary design analysis requires multidisciplinary interaction between different parts of a system (e.g. subsystems). Exchanging data between different disciplines can be a hard task given the lack of standard interfaces. Fundamentally, there are two types of integration of disciplines and tools, described below.

Interface centric $(I C)$, where the models of a specific domains run in the domainspecific tools. The tools are then connected together to simulate systems' behavior. This approach is a great advantage when dealing with high fidelity models and tools. The CAD centric approach is a very well-known type of interface centric approach where CAD initiate the process of multidisciplinary analysis by feeding other design tools either as raw geometry used for example for CFD and FE analysis or as dimensions or mass properties (for instance in dynamic simulation) (Hwang and Martins, 2012; Welle et al., 2012).

Data centric $(D C)$, where all design activity should take place in the same design tool. The output of the tools is reformatted to a neutral model that is readable by all tools. Functional mockup interface (FMI) is an example of handling the exchangeable information. FMI is an evolving tool-independent standard for model exchange and cosimulation. For more information, see (Functional Mock-up Interface, 2016).

The Interface centric (IC) approach has proven to be promising for wide use in the MDO frameworks. This is mainly due to greater experience and familiarity on the part of the engineers with IC accessibility and advances in tools to support IC, fewer changes in traditional design approaches and easier implementation of IC. 
There are many commercial tools on the market to support an IC approach, for example modeFrontier (Esteco, 2016), SORCER (Sorcer, 2016), HEEDS (Red Cedar Technology, 2016), OpenMDAO (openMDAO, 2016) and iSIGHT (iSIGHT, 2016). In the present project, modeFrontier has been used to integrate the models, set up, and run the optimization.

\subsubsection{Complex product design}

The design of complex products is a concurrent collaborative process that involves organizational, management and technological challenges. A complex product consists of a number of coupled systems and subsystems from multiple disciplines. To design a complex product holistically, the distributed heterogeneous models and tools need to be integrated efficiently to represent the product as a whole (Bhise, 2013). System decomposition is used to facilitate the disciplinary modeling (Zhang et al., 2007). Development engineers are assigned to each design problem that may represent a discipline of the multidisciplinary system (Eppinger, 1997; Ulrich and Eppinger, 2016). The disciplinary models are then integrated to demonstrate system interactions, see figure 2-4. As mentioned earlier, multidisciplinary design has proven to be an appropriate method to handle the complexity of designing a complex product.

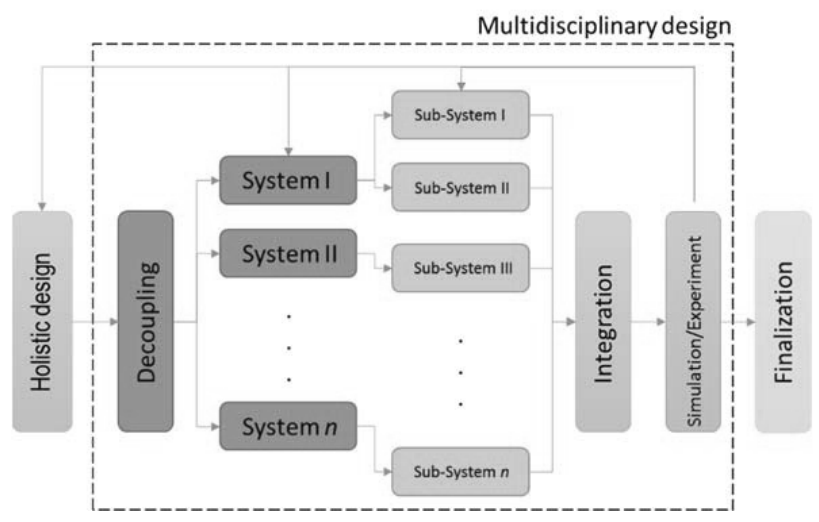

Figure 2-4: Design process of a complex product (adapted from Eppiger, 1995).

\subsection{Multidisciplinary design optimization}

Typically, employing high fidelity models increases the dimension of the design space drastically. As discussed earlier, design optimization is defined to deal with such expanded design space and search to determine the design parameters that lead to optimal design with respect to the predefined design constraint/s. The formulation of a typical design optimization problem can be represented as in equation (1), where depending on the 
number of design parameters and type of the problem, if it is a single objective or multiple objectives, the size of the vectors $\mathbf{x}$ (design vector) and $\boldsymbol{F}$ (objectives vector) varies respectively (Hwang, 1980; Steuer et al., 1986).

$$
\begin{array}{lll}
\min & \mathbf{F}(\mathbf{x}) \\
\text { s.t. } & \mathbf{g}(\mathbf{x}) \leq 0 \\
& \mathbf{h}(\mathbf{x})=0 \\
& x_{i, L B} \leq x_{i} \leq x_{i, U B} & \\
\text { where } & \mathbf{F}=\left[\begin{array}{llll}
f_{1}(\mathbf{x}) & \ldots & f_{z}(\mathbf{x})
\end{array}\right]^{\mathrm{T}} & \\
& \mathbf{x}=\left[\begin{array}{lllll}
x_{1} & \ldots & x_{i} & \ldots & x_{n}
\end{array}\right]^{\mathrm{T}} & \text { Inequality constraints } \\
&
\end{array}
$$

Objective/s and constraint/s in an MDO problem are provided by the interaction of various disciplines. However, a multidisciplinary and complex system, from design optimization perspectives, have to be treated as a united system rather than developing each subsystem independently (Chapman and Pinfold, 2001). MDO helps to mathematically suggest a path in the design space from initial design to optimal design (with respect to objectives and constraints). Large numbers of variables and objective functions are treated simultaneously in an MDO framework - far beyond the power of the human mind. However, it should be considered that MDO is not used to remove the engineers from the design process but rather helps to conduct trade studies.

\subsubsection{MDO fundamentals}

The definition of MDO varies with respect to the requirements, benefits and techniques. Several quotes are therefore presented here to cover the definition of MDO from various aspects.

MDO is defined by Martins and Lambe (2013) as " $a$ field of research that studies the application of numerical optimization techniques to the design of engineering systems involving multiple disciplines or components". The AIAA Technical Committee on MDO (1998) has outlined MDO as "How to decide what to change, and to what extent to change it, when everything influences everything else."

As what can be interpreted from the above definitions, MDO helps to optimize the design of a coupled system. A system is considered to be a coupled system when the inputs and outputs or the design variables or objectives are shared between a number of different disciplines. To facilitate the design of a coupled system, the various disciplines need to be considered concurrently. This means that the various design alternatives should be explored at the same time where the effects of changes in a subset of the design variables are reflected on all the others (Riccardi, 2012). Figure 2.5 gives an overview of a general MDO framework. 


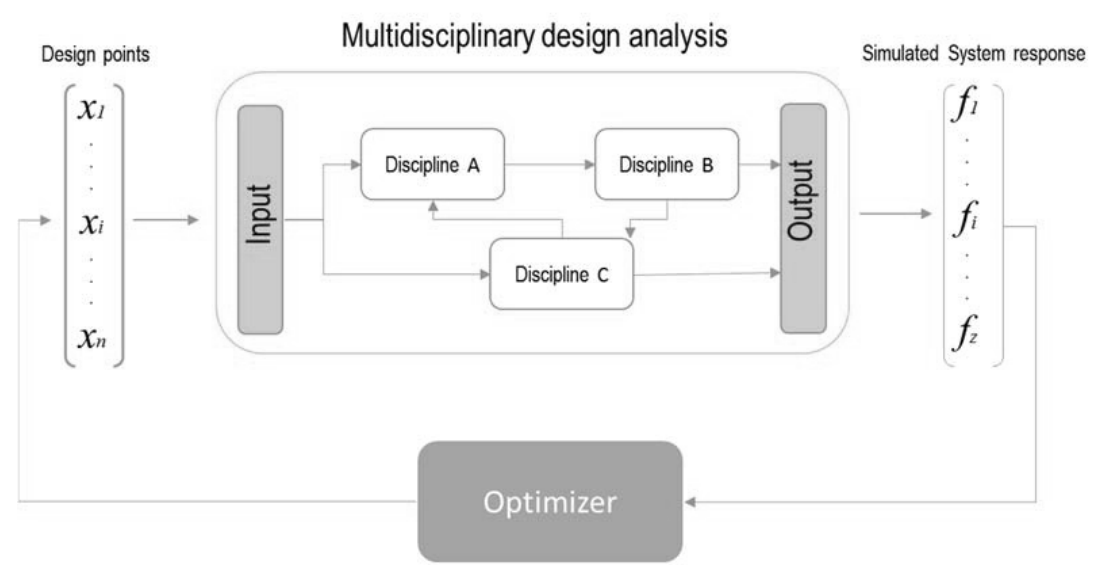

Figure 2-5: A general MDO framework (adapted from Vandenbrande et. al., 2006).

\subsubsection{MDO in conceptual design}

Typically, conceptual engineers are searching for the global optimality of the concept under evaluation (Arora, 2011). The sequential design method (conventional technique to design the multidisciplinary products) is defined where a solution is made through a manual iteration among the individual disciplinary models (Sobieszczanski-Sobieski, 1989). In this method, the shared design variables (coupled variables) between the disciplines are not considered in the analysis, which leads to shrinking the design space and missing the chance to obtain global optimality (Du and Chen, 2004). Typically, sub-optimal design is the best achievement of this approach. Concurrent acting on all disciplines is however a basic feature of an MDO frameworks, which resulted in an extended design space and consequently enhanced the possibility to find the global optimal design (Tarkian, 2012; Amadori, 2012).

\subsubsection{MDO assignments}

An MDO problem can be divided into two sub-problems: multidisciplinary design and design optimization problems. Each of the subcategories consists of many features that need to be clarified before implementation. These are considered in this section to be MDO assignments.

\section{Design optimization problem}

This section deals with the important features that need to be considered to accurately set up a design optimization problem.

- Global vs local optimization: Local optimum is a point in a region where the point is smaller than all the other points in that region (considering a 
minimization problem). However, the global optimum is a point which is smaller than all the other feasible points in the whole design space. A function might thus have a number of local optima in the design space but just one global optimal point (or more points with the same function value). Many optimization algorithms can just guarantee a local optimum, whereas the global optima are sought after.

- Single-vs multi-objective problem: A problem is called single-objective when the aim is to find the "best" solution for one targeted objective. This could be achieved by calculating the value of one objective or lumping many objective functions into one objective. By contrast, a multi-objective problem is a problem consisting of multiple conflicting objectives with no single overall solution. In this kind of problem, the interaction between various objectives presented as a set of compromise solutions or Pareto-optimal solutions (Savic, 2002; Deb, 2001). In reality and industrial practice, most problems are multi-objective.

- Discrete vs continuous variables: a continuous variable is defined where a variable can take on any value between two specified values (the variable bounds); otherwise, it is defined as a discrete variable. For example, in a gearbox, the number of gears is a discrete variable and the speed of a gear is a continuous variable (Nocedal \& Wright, 2006). In complex product development we typically phase a mixture of variables, which puts demands on the algorithms used to solve the problem.

- Linear or nonlinear problem: A problem is considered linear when the output and inputs have a linear relationship. If the power of such an equation is more than 1 , however, the problem is called nonlinear. Correlations (between inputs and outputs) in a complex product are normally in nonlinear form (Nocedal \& Wright 2006), which limits the choice of optimization algorithm.

- Optimization algorithms: An optimization routine is used to search for the best solution in a given design space. In fact, optimization algorithms are used to automate the iterative and time-consuming process toward finding optimal designs by changing the design variables in the MDO framework. The characteristics and features of the design problem, explained above, indicate which type of optimization algorithm is best fitted to the specific problem. Technically, numerical optimization algorithms are classified into two main groups: gradient and non-gradient. Gradient-based methods are normally used when the gradient of the function is easily accessible and calculable. The basic requirement of a gradient-based method is thus the existence of a mathematical equation of the problem in hand and the presence of continuous first order derivatives of such an equation. However, in a model-based optimization approach, generating a mathematical equation that could represent the complex behaviours of the models is difficult (if not impossible). Considering the fact 
that the result of the models is normally presented as values, non-gradient-based methods are the common choice for such problems in industry although they are more computationally expensive than gradient-based methods since they use objective function evaluation to find the optima instead of obtaining Hessian and gradient information of the objective function. Multi-objective genetic algorithms (MOGA), which are classified as non-gradient based methods, have proven to be very helpful in complex industrial applications to optimize multiobjective and multidisciplinary problems (Deb, 2001; Coello et al., 2002; Nosratollahi et al., 2010; Persson, 2015). MOGA has therefore been used in most of the applications in this thesis.

MOGA is a version of GA modified to handle multi-objective problems (Coello et al., 2002). Genetic algorithms are developed based on the mechanism of natural selection (Goldberg, 1989). Each design parameter active in the optimization is coded into a gene. A possible solution consists of all design parameters, forming a chromosome represented as an individual. Depending on the problem at hand, a chromosome could be an array of real numbers, a binary string or a list of components in a database. A set of individuals forms a population. All the individuals in each population are evaluated in the first iteration and the fittest are selected for mating. Mating is performed by combining genes from different parents to produce a child, called a crossover. Mutations may also accrue to produce a unique child. Finally, the children are inserted into the population to form a new generation. This process is repeated in each iteration until convergence is achieved or the predefined maximum number of generations is reached.

\section{Multidisciplinary design}

MDO techniques have been developed extensively over the last two decades to integrate the disciplinary models into a single optimization framework efficiently. Three main topics that can be shared in any MDO frameworks are problem formulation, system decomposition, and MDO architectures:

\section{- Problem formulation}

Several multidisciplinary approaches have been proposed for how to efficiently integrate several disciplines into a single design optimization environment. There are mainly three formulations of MDO problems identified in the literature such as multi-disciplinary feasible (MDF), individual disciplinary feasible (IDF) and all at once (AAO) (Martins and Lambe, 2013).

$\boldsymbol{M D F}$ is the most straightforward definition of an MDO formulation. In this approach, only the design variables are managed by the optimization 
algorithm. A complete multidisciplinary design analysis is performed in each iteration. This ensures the calculation of all coupled disciplines. Hence, in each iteration, complete multidisciplinary feasibility is guaranteed.

$\boldsymbol{I D F}$ is defined in contradiction to MDF where just individual disciplines' feasibility is ensured in each iteration. The optimizer controls the interdisciplinary design variable in each discipline and goes through all the disciplines in each iteration. The coupled variable, which is shared by two or more disciplines, should be duplicated. The main advantage of IDF compared to MDF is higher computational efficiency, and easier to modify or add disciplines. However, the IDF may lose efficiency when the number of coupled parameters increases.

$\boldsymbol{A} \boldsymbol{A} \boldsymbol{O}$ is defined where the optimizer controls all design parameters and disciplinary outputs including behavioral variables. Although the number of optimization variables is significantly increased and the optimization problem become more complex, no design solution may be lost in the optimization process. A drawback of this approach is that most of the disciplinary models should be recreated to provide all design variables for the optimization algorithm. Few applications of this method have therefore been reported in the literature (Riccardi, 2012).

- System decomposition

System decomposition is defined as a beneficial method to facilitate the process of multidisciplinary analysis by subdividing the system into smaller, less complex parts (subsystems). System decomposition methods can be classified into hierarchical and non-hierarchical (Sobieszczanski-Sobieski, 1989), see figure 2-6.

- Hierarchical system decomposition (HSD) applies if the system can be divided into a set of modules forming a precise hierarchy. Each part (subsystem) requires information from higher level and there is no information sharing at the same decomposition level. This facilitates the parallel analysis and optimization of each subsystems group.

- Non-hierarchical system decomposition (NHSD) applies if the system is fully coupled and cannot be decomposed hierarchically. In this case, the problem should be considered as a whole and information should be exchanged also in the lateral direction. This makes the optimization problem more complex. 

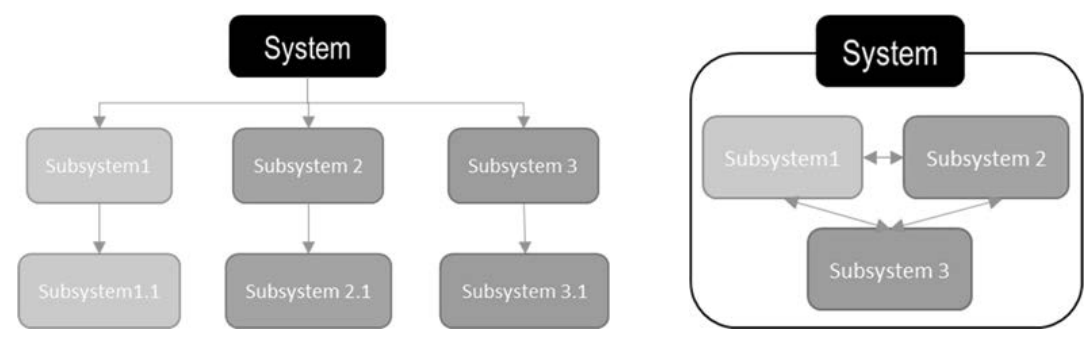

Figure 2-6: Examples of hierarchical (left) and non-hierarchical (right) system decomposition.

- MDO architecture

Similar to problem decomposition, MDO architecture deals with the structure of the multidisciplinary analysis and optimization rather than MDO problem formulation. An MDO architecture can therefore be classified for both HSD or NHSD and as MDF, IDF or AAO. A number of MDO architectures are proposed in the literature such as Black-Box optimization (Jones et al., 1998), which mainly deals with MDF and non-decomposable systems, Single level and multi-level optimization (Yi et al., 2008), Nested optimization loop, concurrent subspace optimization (Sobieszczanski-Sobieski, 1989), Bi-level optimization system synthesis (Sobieszczanski-Sobieski et al., 1998; Sobieszczanski-Sobieski et al., 2000), and collaborative optimization (Kroo et al., 1994; Braun and Kroo, 1995; Braun, 1996).

\subsubsection{Efficient computing}

Time is an important factor in conceptual design. A conceptual engineer needs to evaluate different design concepts and choose the optimum design rapidly without halting the design process.

A remedy to integrate HFMs in conceptual design efficiently is to employ computationally efficient models early in the optimization process. If these models reveal that the design concept is not feasible, the analytical process can be terminated to save time. Another remedy is to use sensitivity analysis before optimization to determine the influence of input parameters on the objectives. This may result in a reduction in the number of design parameters and consequently fewer changes to the design concept in each iteration and therefore faster analysis.

A popular alternative approach is to replace computationally expensive models with numerically efficient models called surrogate models (Forrester et al., 2008; Myers and Montgomery, 1995; Queipo et al., 2005). 


\section{Sensitivity analysis}

Sensitivity analysis can be used both before and after optimization to determine the correlation between the design parameters and objectives or to test the robustness of the objective function to the changes.

Different techniques can be used to perform sensitivity analysis such as local, one at a time, and global sensitivity analysis (Saltelli et al., 2008). Global sensitivity analysis (GSA) as described by Saltelli et al. (2000) is very suitable approach where the design is multidisciplinary and complex and formulating the objectives (or the partial derivatives) is non-trivial and the design space is large. It is often implemented using Monte Carlo techniques, where a representative (global) set of samples is used to explore the design space. The objective function is evaluated at each combination of parameter values input to multidisciplinary coupled system simulation (Saltelli et al., 2008).

\section{Surrogate modeling}

Surrogate models, or meta-models, are approximate models which are numerically efficient and can mimic the behavior of the system in a given design space (Myers et al., 2009).

A surrogate model is created by first generating samples in the design space and performing experiments or simulations of the system. The surrogate model is then fitted to the samples using different methods, e.g. Anisotropic Kriging (AK) (Martins et al., 2005).

The accuracy of the surrogate model is highly dependent on an efficient sampling and fitting method. The number of samples or design of experiments (DOEs) and their placement over the design space have great impact on the accuracy of a surrogate model. Uniform Latin Hypercube sampling (ULH) is used in the present work to create the samples on the design space (Mckay et al., 1979). This method has proven to be advantageous for similar purposes in other researchers' work (Tarkian, 2012; Persson, 2012). For more information regarding relevant sampling methods, see (Wang et al., 2002; Persson, 2012; Myers et al., 2009).

Anisotropic Kriging is a modified version of Kriging that calculates the new desired point as a function of distance to the known point (Martins et al., 2005). The function is resolved by analysing the model output values varying in the design space and in different directions (Pebesma et al., 1998).

\subsection{Collaborative design}

Collaborative design is described by Wang et al. (2002), as "a design process where a product is designed through the collective and joint efforts of many designers". Collaborative design is also referred to as concurrent design or interdisciplinary design (Wang et al., 2002). 
Engineering design of complex products is a collaborative activity that involves multiple experts and teams. The working principles behind each system in a complex product involve a multitude of engineering disciplines. A straightforward method to control and manage the complexity of conceptual system engineering is therefore collaborative design (Kvan, 2000; Peng, 1994).

The concept of collaborative design has been extensively studied from different perspectives such as organizational (Kolfschoten and De Vreede, 2007), process (Fähling et al., 2011), technical (Favela et al., 1993; Eynard et al., 2005; Paternò, 2001; Karlsson et al., 2005), and even from a sociotechnical perspective (Lu and Cai, 2001). Although the collaborative design has been developed from different perspectives, as mentioned by Klein et al., (2006), "current collaborative design approaches are as a result typically characterized by heavy reliance on expensive and time-consuming processes, poor incorporation of some important design concerns (typically later life-cycle issues such as environmental impact), as well as reduced creativity due to the tendency to incrementally modify known successful designs rather than explore radically different and potentially superior ones".

Haake et al. (2010) and Lukosch and Kolfschoten (2011) have investigated different possible challenges that may affect the efficiency of an engineering collabaotive design activity and gategorized them into group level and process level challenges. Group level challenges mainly concern defining the roles and interaction inside the group. Process level challenges mainly concern free riding, supremacy and hidden agendas.

Another challenge in collaborative engineering design is to make a design decision that is acceptable to all engineers and could satisfy interdependencies of the concept (Kodiyalam and Sobieszczanski-Sobieski, 2002). As mentioned earlier, MDO has proven to be useful to find such compromises and propose the best design concept accordingly. However, developing a MDO framework requires collaboration among the design team (Simpson and Martins, 2011; Agte et al., 2009).

Conceptual engineers are traditionally responsible for exploring the design space and finding creative solutions. However, conceptual engineers require more detailed information to be able to reasonably choose the best concept (Howard et al., 2007). This information is normally created in the detail design phase, where the domain experts work. Domain experts develop high fidelity engineered subsystems. In the present work, therefore, the definition of collaborative design is simplified and defined for a specific kind of simultaneous collaboration between conceptual engineers and domain experts. The other types of collaboration are also needed for successful development of complex products; in this thesis, however, the specific collaboration between conceptual engineers and domain experts is in focus (Uflacker and Zeier, 2011). 
Direct, tight collaboration between conceptual engineers and domain experts can reduce the cycle time in the development of complex systems (La Rocca et al., 2007). This approach brings forth many advantages, some of which are described below:

- Domain experts can develop models that are simple enough to be used in a conceptual study and still reflect the performance characteristics of an actual system.

- Verification and validation of models are important tasks. Engineers proficient in their profession are able to conduct verification and validation tasks more effectively whereas conceptual engineers may not be equipped with the resources to verify the models themselves (Steinkellner, 2011).

- Domain experts have in-depth understanding of the nature of their domains and can better estimate parameters that are used to predict system and component properties. They can also estimate technology trends that can be incorporated into the models (La Rocca et al., 2011; Larson and Wertz, 1999).

Nowadays, with a more computerized design process, collaborative design can be made even more effective. There are a large number of collaborative tools and methods that allow simultaneous work on complex systems, reduce manual and sequential operations, and ultimately speed up the design process, e.g. web service technology, Product Lifecycle Management (PLM) systems, enterprise-wide collaboration platforms, intelligent personal assistant (IPA) using knowledge based engineering as well as simpler tools like Dropbox (Törlind and Larsson, 2002; Danahy et al. 2012; Pokojski, 2006). However, the detailed collaboration between conceptual engineers and domain experts is not explicitly supported by these techniques.

This thesis tries to bring out the benefits of employing domain expert knowledge represented as HFMs in conceptual design - as an important phase in the engineering design process - and boosts the products' knowledge foundations. Collaborative design is key to facilitating the collaboration between concept engineers and domain experts. The thesis concentrates on complex and multidisciplinary products where the problems are multi-objective and nonlinear, with the combination of discrete and continuous variables. MDO is therefore used with the help of non-derivatives optimization algorithms (e.g. MOGA: s) to optimize the product. Hierarchical decomposition is used to simplify the model development as well as the design collaboration. Finally, computationally efficient surrogate models are employed to reduce the design and optimization time (as it is a vital factor in conceptual design). 


\section{3 \\ Contributions}

E

MPLOYING high fidelity models in the conceptual design of complex products has proven to be very valuable in increasing the knowledge foundation and illuminating the unknown-unknown part of the design (Loch et al., 2006). However, development and integration of HFMs into conceptual design require close collaboration among the design team. The main contributions of this thesis are therefore to define and develop the CMDO framework, including a set of defined roles and tasks, collaboration requirements, and a process for easy implementations of tasks. A step-bystep guideline is therefore presented to MDO practitioners in order to facilitate the implementation of CMDO in an engineering team. The helpfulness and benefits as well as difficulties and challenges of implementation of the proposed framework are evaluated in various case studies and application examples.

The idea of CMDO has been evolved step by step and over the course of this research where each case study contributes to evaluate and improve the concept. The case studies are extensively discussed in the appended papers. To cut a long story short, this chapter will therefor concentrate on the final, improved version of the CMDO framework and discuss its main elements. For more information about the development process and the application examples, the reader is referred to the appended papers II, III, IV and V.

In this chapter, the components of the CMDO framework are presented firstly in terms of roles and tasks and required processes. A guideline for implementing a CMDO framework in a large engineering team is then presented. The chapter concludes with an application example of a tidal water power plant. 


\subsection{Collaborative multidisciplinary design optimization (CMDO)}

CMDO tackles the complexity of implementing MDO for conceptual design of complex systems in a setting of large engineering teams. The idea behind CMDO is to bring the domain experts' knowledge into the conceptual design either directly (in the decisionmaking process) or indirectly (by bringing HFMs into conceptual design). Many technical and collaborative tasks need to be completed to develop a CMDO framework. A CMDO framework consists of two main elements: CMDO tasks and the responsibility to perform the tasks expressed as CMDO roles.

\subsubsection{Roles and responsibilities in a CMDO framework}

A number of complex multidisciplinary tasks are involved in a CMDO framework and obviously the conceptual engineer is not able to take sole responsibility for performing all of them. For this reason, CMDO is constructed in a joint effort between different engineers and engineering disciplines. Four roles are outlined as having the main responsibility for performing the CMDO tasks, see figure 3-1. They are defined as:

- Conceptual Engineer (CE)

- Domain Expert (DE)

- Interface Expert (IE)

- Optimization Expert (OE)

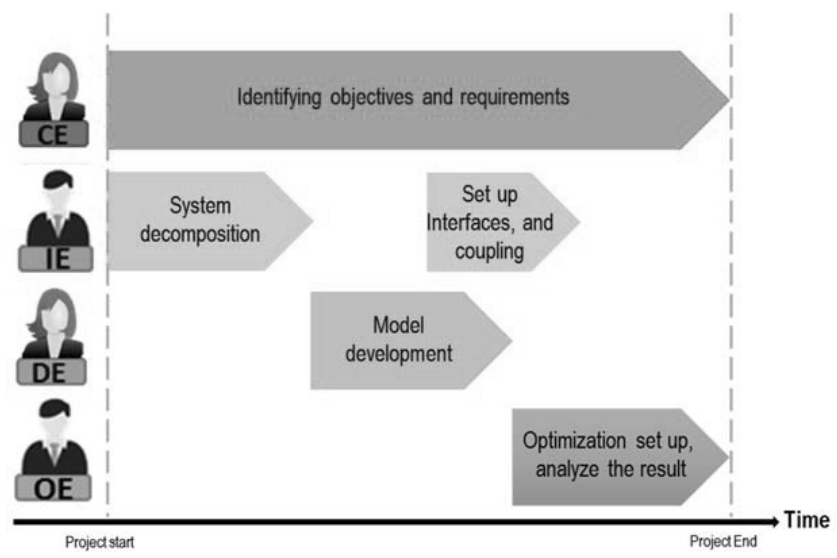

Figure 3-1: Roles and main responsibilities in a CMDO framework.

$\boldsymbol{C E}$ is an engineer who can decide on the features of a new concept in order to fulfill customer requirements and preferences. They can also propose suitable approaches to evaluate the concepts in terms of quality or performance. In fact, the CE defines the preliminary objectives and constraints of the product and must therefore have a good 
overview of the product requirements in terms of complexity and preferences. The $\mathrm{CE}$ must also have a generic understanding of the product or system being designed.

$\boldsymbol{D E}$ is a skilled engineer who is a specialist in his/her domain or discipline. A DE should be familiar with the area that the new concept is to be developed for. He or she should also be aware of the limitations and restrictions they may be faced with when developing the new concept, e.g. regarding the limitations of design software and hardware tools.

$\boldsymbol{I E}$ is an experienced engineer with a deep understanding from different design departments within a company. The IE should be able to act as project manager to assemble the team and should therefore have a good overview of the capabilities and limitations of each department and discipline. In addition, a deep knowledge of how different disciplines may or may not work together is crucial to the IE role.

$\boldsymbol{O E}$ is an engineer who is able to formulate the problem, run the optimization, extract and analyze the results of the optimization, and present and illustrate the results in a way that facilitates the decision-making process performed mainly by the $\mathrm{CE}$.

The above roles could be assigned to a team or an individual engineer. There is also the possibility for one engineer or a team to take on multiple roles. However, due to the complexity of the involved tasks and special proficiency required to perform the tasks, taking multiple roles has proven to not be so efficient.

\subsubsection{CMDO Tasks}

There are some series of tasks involved in a CMDO framework, which have been categorized into four main blocks:

- Object identification and system decomposition

- Model development and evaluation

- Model integration and multidisciplinary assessment

- Optimization and efficient computing

All the above blocks consist of multiple subgroups, each including a number of tasks that need to be performed to develop a CMDO framework. The above-mentioned tasks are described below together with the roles that are responsible for performing them. An illustration of the blocks, roles, and responsibilities is also shown in figure 3-2.

\section{Object identification and System decomposition}

The design objectives, requirements, and limitations are mainly defined by the CE. The CE should also define a number of important design parameters from customer requirements' perspectives. The objectives (OBs) can be both technical and business OBs, e.g. cost and profit. Typically, OBs in conceptual design are defined using a top- 
down approach. This means that the objectives on high hierarchy levels (e.g. product level) are defined first and should be prioritized in relation to the objectives on lower hierarchy levels (e.g. the sub-systems and component-related objectives). On the other hand, the DE could bring disciplinary perspectives regarding the influence of each discipline on the defined objectives. Although the $\mathrm{CE}$ has the main responsibility to define OBs, the collaboration between the $\mathrm{CE}$ and $\mathrm{DE}$ may result in a more accurate definition of OBs and design constraints (COs).

In a multidisciplinary product, the defined objectives and constraints (explained above) are normally calculated from interactions between tightly coupled disciplines. Hierarchical System Decomposition (HSD) is used to break the system down into smaller parts that can be analyzed faster and more easily. HSD is also used to facilitate the contribution of domain experts in conceptual design. The IE has the main responsibility for performing the HSD. The OBs and COs need to be taken into account for an efficient system decomposition. In fact, a system is preferably decomposed into smaller parts (subsystems) in such a way that OBs or COs can be provided by subsystems directly. The preliminary data flow between the system, subsystems, and components should also be defined at this stage. A backward approach is used here to map the dataflow easily. The first task in this approach is to establish the OBs and COs. The associated systems, subsystems, and components to provide the selected OBs and CBs are set next. The design parameters are chosen in the last step based on suggestions from the CE or the characteristics of the selected components that can be described by the DE. The data flow is mapped by drawing a line between design parameters and the OBs and COs. The collaboration between the CE, DE and IE facilitate the decomposition process.

\section{Model development and evaluation}

Domain experts (DEs) are responsible for developing or modifying and adapting the domain-specific models suggested by the IE based on the system decomposition. A DE should receive all the requirements and specifications of the design and in particular about disciplinary models from both the $\mathrm{CE}$ and the IE. The limitations and assumptions (L\&A) considered for each model should be decided by the DE considering the suggested data flow. However, such L\&A should be discussed within the design team to ensure that all the requirements are met. Each model's performance should be evaluated within the predefined design space. This process is called Disciplinary Feasible Analysis (DFA) in the guideline and is explained extensively in the appended paper [IV] and Section 3.2.

\section{Model integration and multidisciplinary assessment}

The developed disciplinary models are coupled together to create the suggested data flow. The model's integration can be performed with the help of an integrator software tool as described in Section 2.1.3. Here, modeFrontier (Esteco, 2016) has been used as an integrator and optimizer tool. 
The disciplinary models integrated in the framework can behave differently due to new requirements and limitations arise as a result of the coupling. The coupled models need to be assessed before optimization. Multidisciplinary feasibility analysis (MFA) is defined as a process to facilitate the mentioned assessment process. For more information, see the appended paper [IV] and Section 3.2.

\section{Optimization and efficient computing}

The optimization expert (OE) is responsible for formulating the problem based on the data flow and additional information provided by the $\mathrm{CE}$ and $\mathrm{DE}$ regarding the priorities, requirements, limitations, and behavior of the models, etc. Based on the problem formulation, the OE should set up the optimization, including the selection of proper optimization algorithm and algorithm-specific parameters. The problems in this study are normally classified as multi-objective problems and algorithms that can handle multi-objective problems such as multi-objective genetic algorithm (MOGA) or Complex (Krus et al., 2003; Krus et al., 2013) are therefore used. modeFrontier (Esteco, 2016) is used as a tool to handle both tool integration and optimization in almost all the case studies presented in the appended papers.

The computational efficiency of the MDO process is very important, in particular in conceptual design. The OE is responsible for ensuring such efficiency. Here, two methods are used to speed up the optimization process. First, the computationally efficient models are placed early in the optimization. If such models reveal that the design is not feasible, there is no need to run computationally heavy models. Second, the computationally heavy models are replaced with surrogate models. The design points are created using Uniform Latin Hypercube (ULH) due to its proven benefit for similar design cases (Tarkian, 2012; Persson, 2015). Anisotropic Kriging (AK) as one of the proper fitting method, suggested for models with complex behavior, is used to create the meta-models (Simpson et al., 2001).

\subsection{A guideline for CMDO implementation}

The CMDO framework, including roles and tasks, has been evaluated in various case studies (presented in appended papers I to IV). The experience gained from each case study, including barriers to and challenges on implementation (as reported in appended papers), is used to develop a step-by-step CMDO implementation guideline. An overview of the proposed guideline is presented in figure. 3-2 and consists of the four blocks described above. 


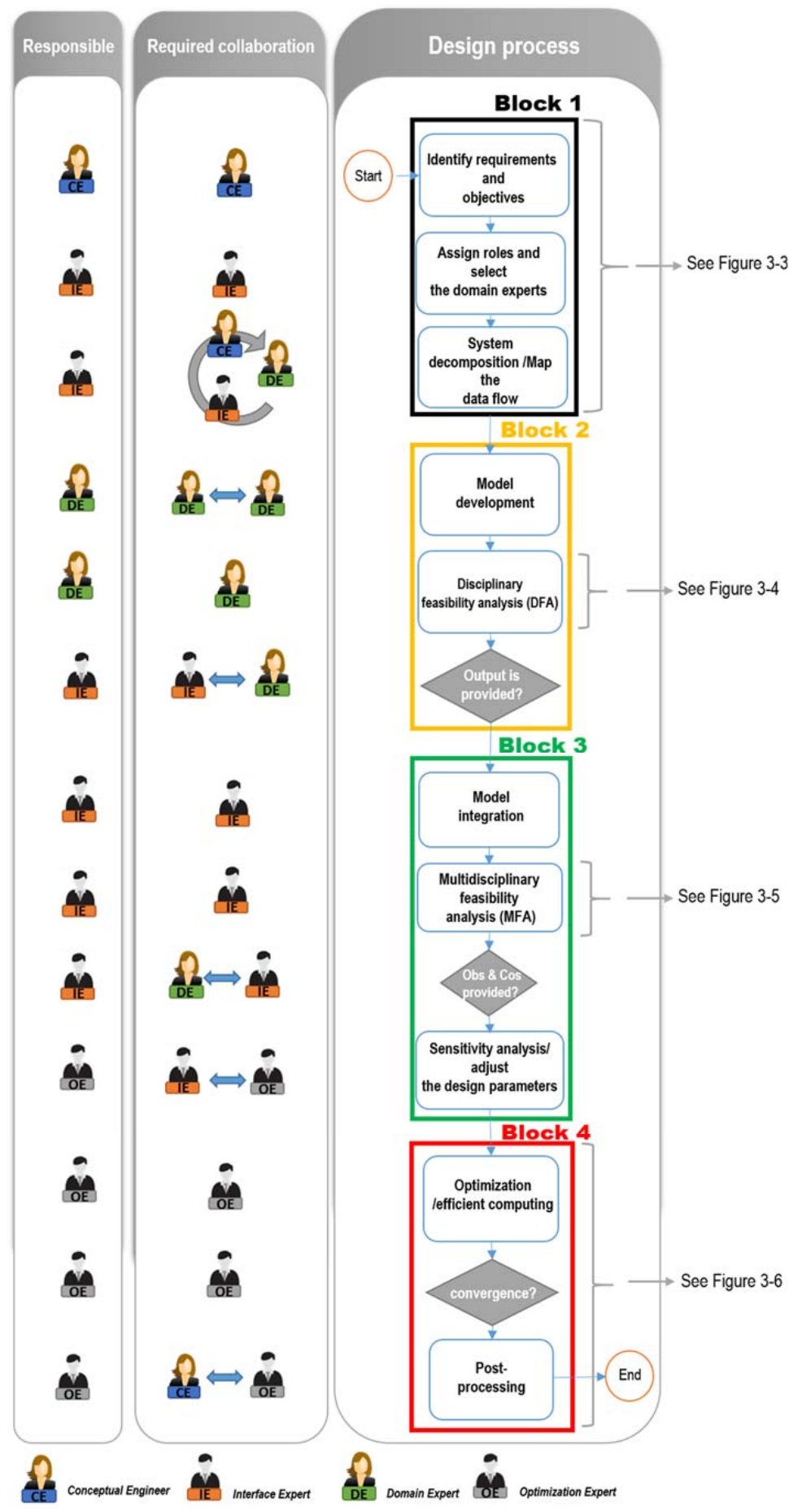

Figure 3-2: Overview of the proposed guideline to create a CMDO framework. 


\section{Block 1}

The first block helps to formulate the problem, form the group, assign the roles, and decompose the system into multiple disciplines. The IE and CE are responsible for performing the tasks in block 1 efficiently. However, the DEs' interdisciplinary knowledge could assist in performing the hierarchical system decomposition (HSD) more reasonably by considering the requirements and limitations of each discipline, see figure 3-3.

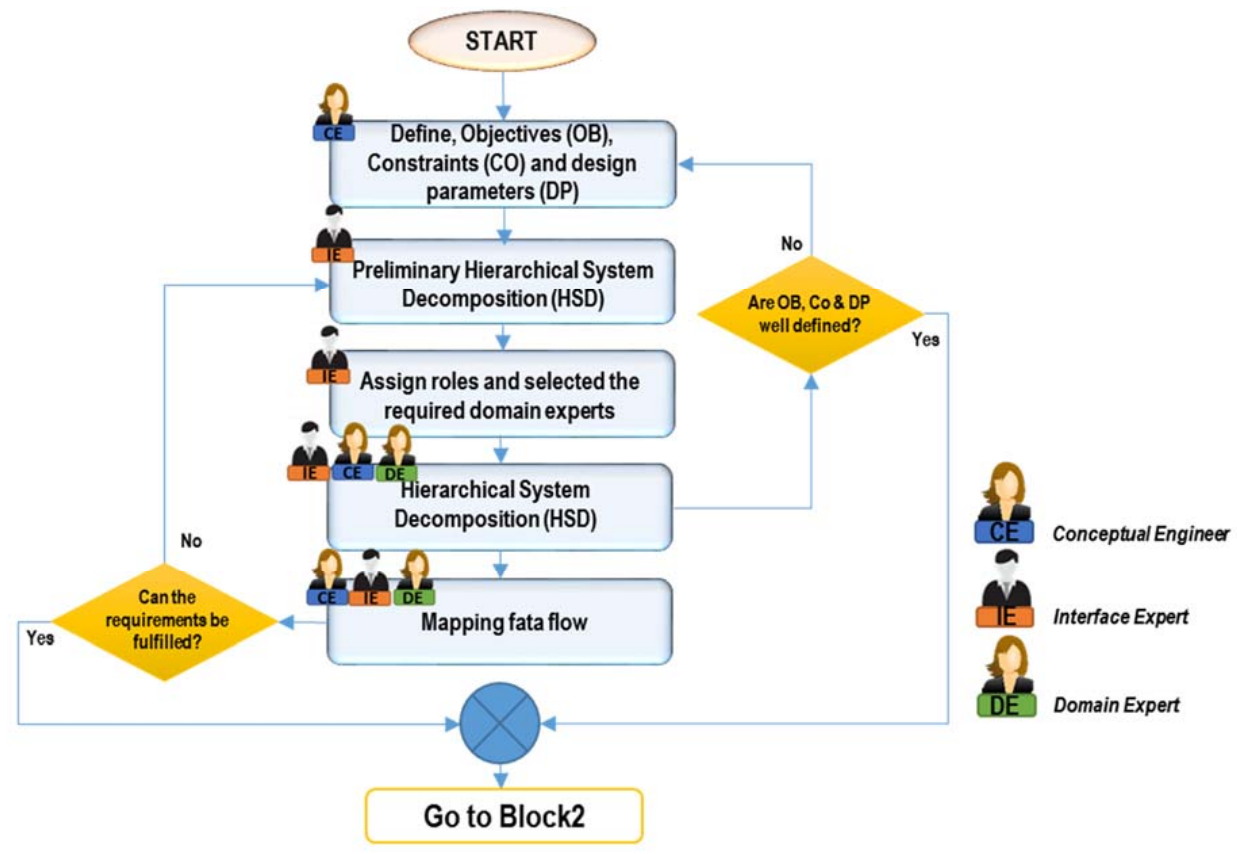

Figure 3-3: Flowchart of performing the tasks of block 1.

\section{Block 2}

The DEs have the main responsibility for the tasks in the second block, which contains model development and disciplinary feasibility analysis. The disciplinary feasibility analysis (DFA) process is explained in figure. 3-4. In this step, a set of random design variables is generated within the design space and fed into the disciplinary models. The performance of each model is evaluated with respect to the data flow suggested in the first block, and the models are checked for feasible output, for more information, see paper [IV]. 


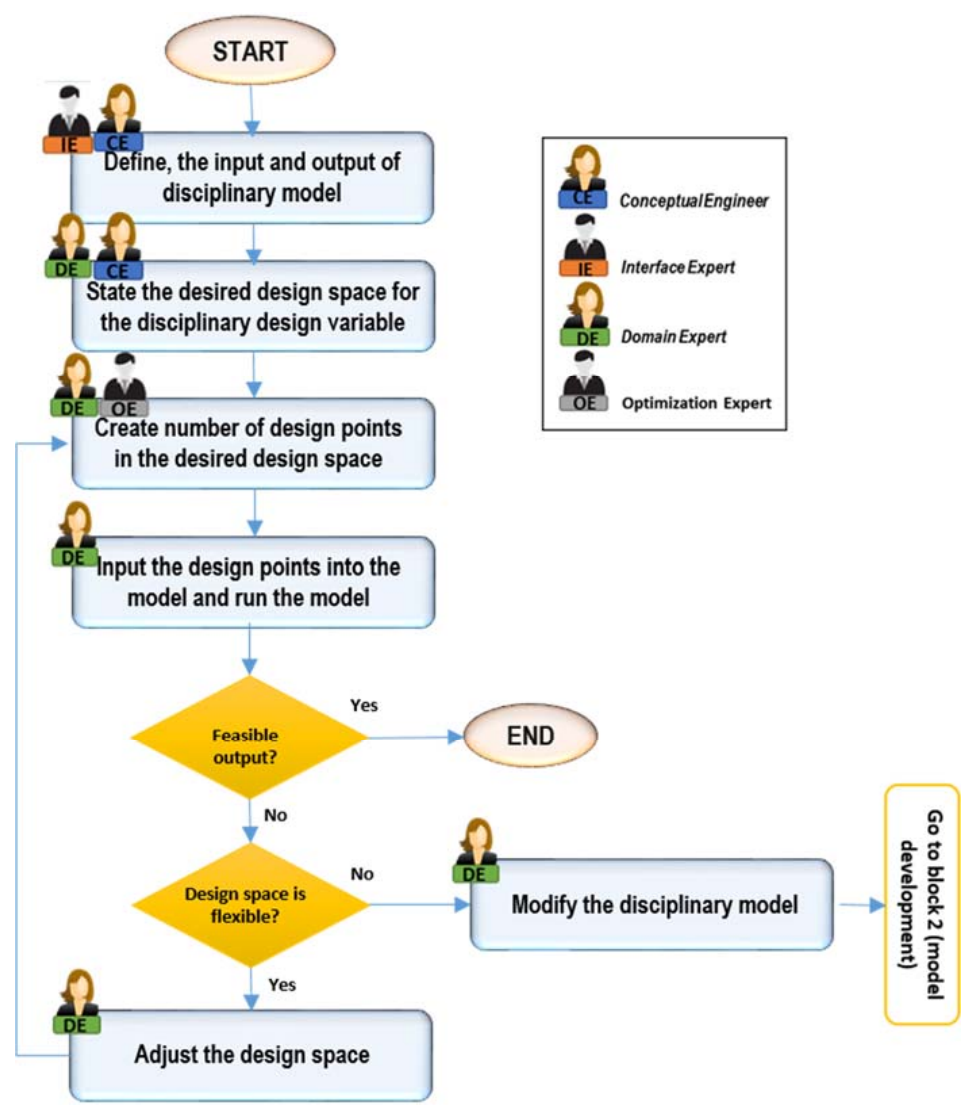

Figure 3-4: Flowchart of performing a disciplinary feasibility analysis (DFA).

\section{Block 3}

The behavior of the disciplinary models may vary when they are coupled together. This is mainly due to the new constraints applied on product level and on data sharing between the models. In block 3, the developed disciplinary models are coupled and assessed with respect to multidisciplinary objectives and constraints. The assessment of the coupled system is done by performing a multidisciplinary feasible analysis (MFA), as described in figure 3-5. The process starts with the creation of sample design points that uniformly cover the design space. The design points are then fed into the coupled multidisciplinary system. The result of the coupled system is investigated and evaluated with respect to the requirements, OBs and COs. If the coupled system could not provide the OBs and COs, the disciplinary models should first be updated and recoupled to each other. However, if such a modification could not solve the problem, the IE should call a full meeting of all the team members and present the problem. A conceivable decision from such meeting might be to modify the constrains or the suggested HSD and data flow. It should be 
remembered, however, that, this is a drastic measure that could affect the efficiency of the whole design process and increase the design time significantly.

The IE plays a central role in performing the MFA efficiently. Collaboration with the CE and DE to evaluate the result could ease the modification process that may ensue.

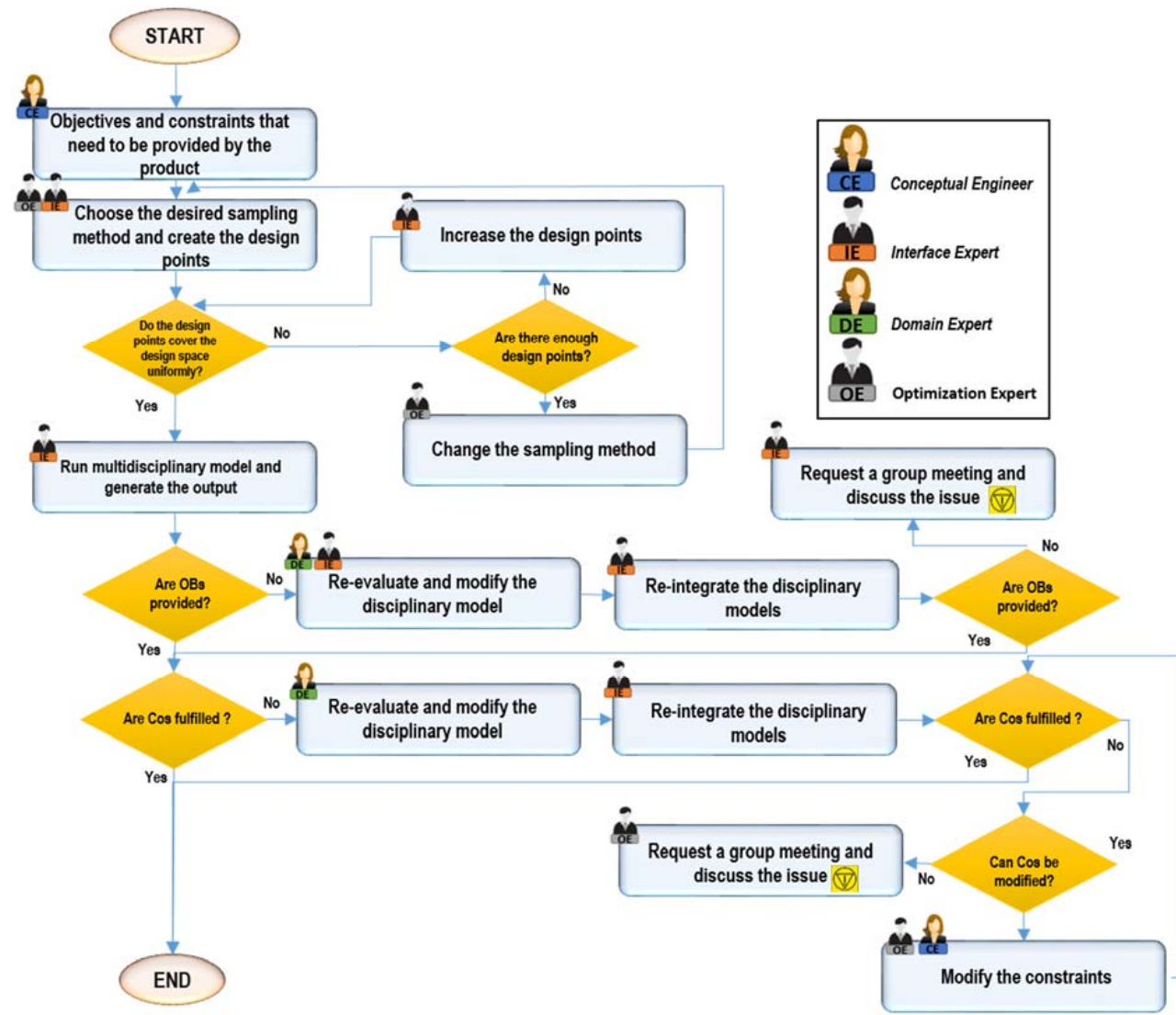

Figure 3-5: Flowchart of performing multidisciplinary feasibility analysis (MFA).

Intelligent selection of design parameters has a direct impact on the performance of the MDO. The effectiveness of the design parameters on the design objectives is therefore evaluated through a sensitivity analysis, presented in the latest stages of Block 3. A sensitivity analysis can be easily achieved by reusing the design points created for the MFA process and it is therefore suggested that the sensitivity analysis be conducted in block 3, prior to running the optimization. The results highlight the correlation between the design objectives, the constraints, and the design parameters. The results can be used to either modify (adjust the variable range) or reduce the dimensions of the design space (remove less effective or ineffective design parameters), which leads to a more efficient computational process. 


\section{Block 4}

Optimization and post-processing are done in Block 4. The OE has main responsibility for performing the tasks in Block 4. The first task is to identify computationally heavy models. As mentioned in chapter 2, one popular choice to speed up the optimization process is to replace the computationally heavy models with surrogate models. A number of design of experiments (DOEs) are needed to create surrogate models. To speed up the process, the design points created for DFA analysis can be used as DOEs to create surrogate models. The computationally heavy models in the coupled multidisciplinary system are then replaced with their associated surrogate models. The OE then formulates the problem mathematically and sets up the optimization. Selection of optimization algorithm including algorithm-specific parameters, optimization architecture (parallel or single level optimization), approaches to treat the constraints (e.g. penalize one or more objectives or consider them as extra objectives) are a few examples of the tasks that the OE needs to consider prior to running the optimization. The OE should keep track of optimization convergence and the accuracy of the results. The post-processing part includes illustration and interpretation of the optimization results, which is mainly done in collaboration between the $\mathrm{OE}$ and the $\mathrm{CE}$ as the end users of the results, see figure. 3.6.

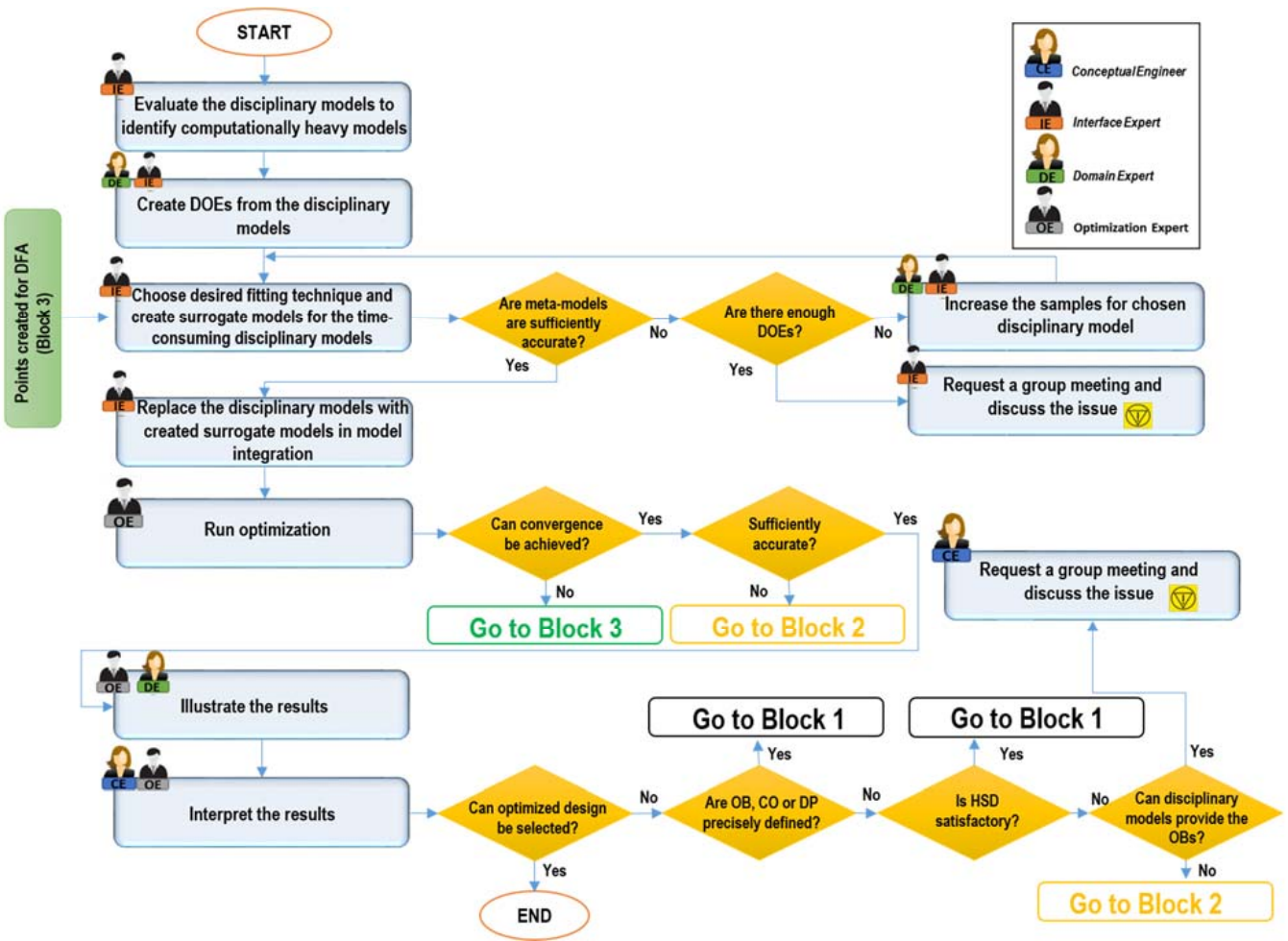

Figure 3-6: optimization and post-processing flowchart (block 4). 


\subsection{Example of CMDO framework}

During this study, the proposed CMDO framework has been implemented successfully in various design applications. One of the design studies that is extensively discussed in the appended papers (III, IV and V) concerns the design and optimization of a novel concept for a tidal power plant called Deep Green, developed by Minesto AB. Deep Green converts renewable energy from tidal and ocean currents into electricity. It consists of a wing (kite) attached to a turbine, where the movement of the kite produces an intake flow that passes through the turbine and generator to produce electricity (for more information see, (Minesto, 2016)). The hierarchical system decomposition (HSD) of the Deep Green concept is shown in figure 3-7. The focus area of this study is the turbine system with the subsystems stator, turbine, and generator. The objective of the study is to optimize the geometry of the turbine system to achieve minimum weight and extract maximum power.

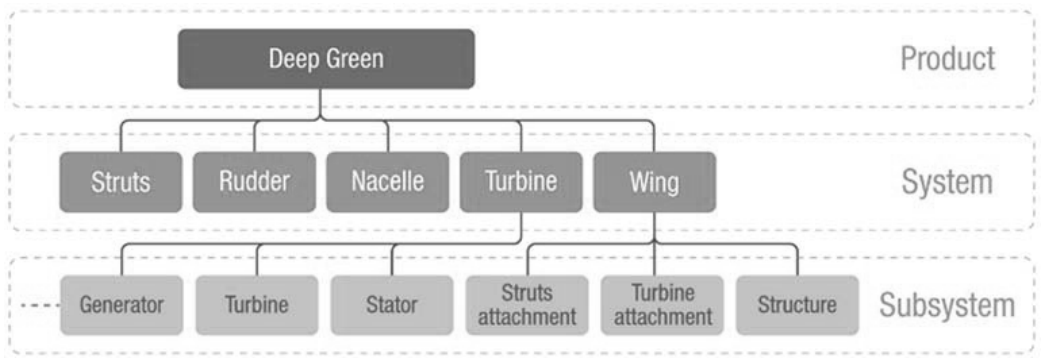

Figure 3-7: Decomposition of the tidal power plant's system.

This project was established through a close collaboration between Minesto AB as an official industrial partner of Linköping University $(\mathrm{LiU})$ and three different divisions at $\mathrm{LiU}$. The industrial partner takes the role of $\mathrm{CE}$ in this context. It also helps the IE to perform its task efficiently. The other roles, are divided between three divisions at LiU: Applied Thermodynamics, Fluid and Mechatronic Systems, and Machine Design. The distribution of the actual MDO tasks between these roles is addressed in appended papers III and V.

Geometric modeling (CAD), computational fluid dynamics (CFD), finite element (FE), and dynamic simulation are considered to be the main disciplines involved in this case study, and hence the four DE roles are spread over individual engineers. Figure 3-8 illustrates the interfaces and data flow between different disciplines in order to fulfill the objectives (min weight \& max power generation) and constraints (max stress). The OBs and COs are proposed primarily by the CE. Seven design variables (combinations of continuous and discrete variables) are involved in this application study, as shown in the table $3-1$. 
36 Contributions

Table 3-1: Optimization variables and their upper and lower limits.

\begin{tabular}{llll}
\hline Optimization variable $(x j)$ & Lower band $\left(x^{L_{j}}\right)$ & Upper band $\left(x^{U_{j}}\right)$ & Unit \\
\hline Generator type & 1 & 10 & - \\
Rotor thickness & 0.4 & 1.3 & $\mathrm{~cm}$ \\
Rotor angle & 6 & 11 & Degree \\
Rotor diameter & 22.5 & 27.5 & $\mathrm{~cm}$ \\
Stator thickness & 0.6 & 1.5 & $\mathrm{~cm}$ \\
Stator angle & 22 & 32 & Degree \\
Number of blades & 9 & 22 & - \\
\hline
\end{tabular}

The disciplinary models shown in Fig 3-8 are developed by the DEs and coupled together to provide the predefined OBs and CO. However, the results of the MFA and sensitivity analysis show that the system is less affected by the constraint. The DEs therefore suggest that the constraint of Eigen frequency needs to be taken into account and added to the optimization formulation. This can be considered one of the preliminary results of the CMDO.

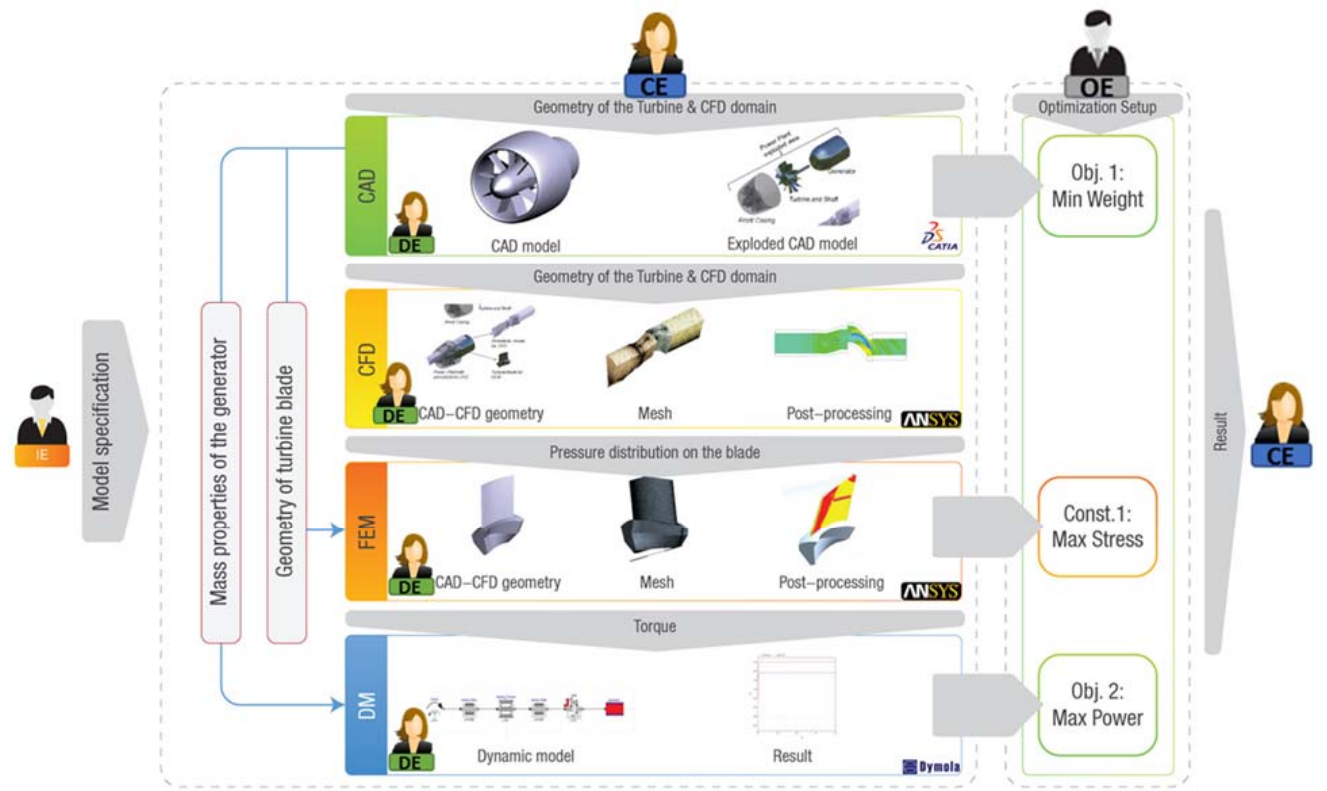

Figure 3-8: Data flow between the different disciplines involved in the CMDO application presented in Paper [III].

$\mathrm{CFD}, \mathrm{CAD}$ and FE analysis are recognized as the most time-consuming models in this study. Surrogate models are therefore created using Anisotropic Kriging and integrated into the framework instead of the real models. The MOGA is used as an optimization algorithm to search the design space. 
The preliminary results of the optimization are shown as a Pareto front, which highlights the effect of the number of rotor blades on the objectives (power and weight), see figure. 3-9. For more information, see appended papers III and V.

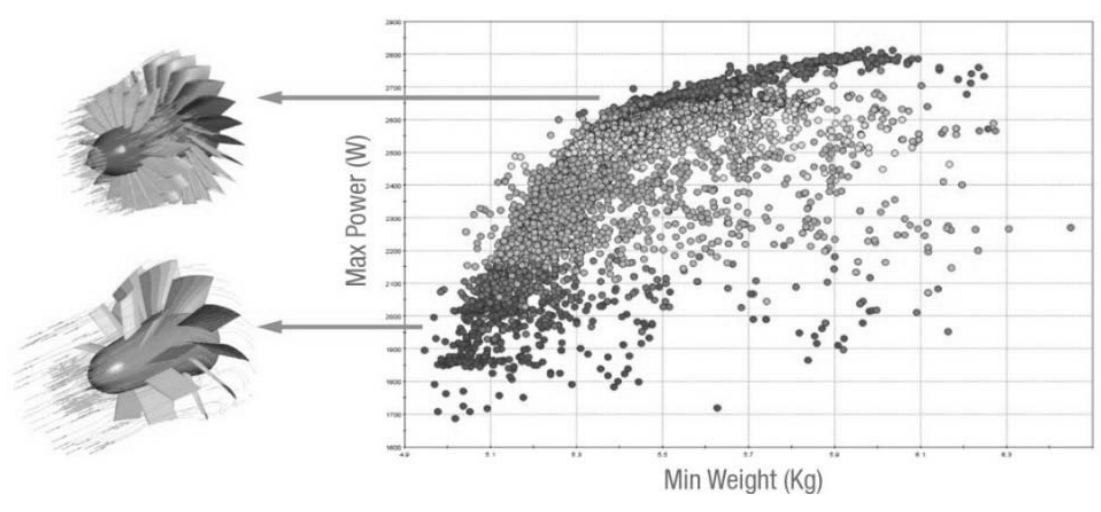

Figure 3-9: Sample results of the optimization presented to the conceptual engineer. 



\section{4}

\section{Summary of the appended papers}

$\mathrm{N}$ addition to Table $1-1$ in section 1.5 , this chapter provides a brief overview of the
appended papers, including main contribution, relation to each other, and how they
support the thesis. For more information, see the appended papers.

\subsection{Paper I: A Collaborative Tool for Conceptual Aircraft Systems Design}

In this paper, the importance of having higher fidelity analysis, (e.g. dynamic simulations) in conceptual design to increase the knowledge foundation is explained. The collaborative design method is presented as a key enabler and facilitator to integrate the HFMs into the CD. A software tool entitled Collaborative Aircraft Vehicle Engineering (CAVE) has been developed to facilitate dealing with HFMs for conceptual engineers. CAVE was developed to assist aircraft systems design but the idea of CAVE is generic and not limited to a specific application.

The dynamic models of the aircraft systems are developed in Dymola and integrated into CAVE. CAVE was developed on a Microsoft Excel platform (using Visual Basic) which most conceptual engineers are familiar with. In fact, CAVE acts as a user interface to facilitate the communication between CEs and HFMs. CAVE is evaluated using a case study from aircraft conceptual system architecture design and optimization. In this study, 3 aircraft systems, i.e. environmental control system, flight control actuation system, and power generation system, are presented and evaluated using a predefined flight profile. 


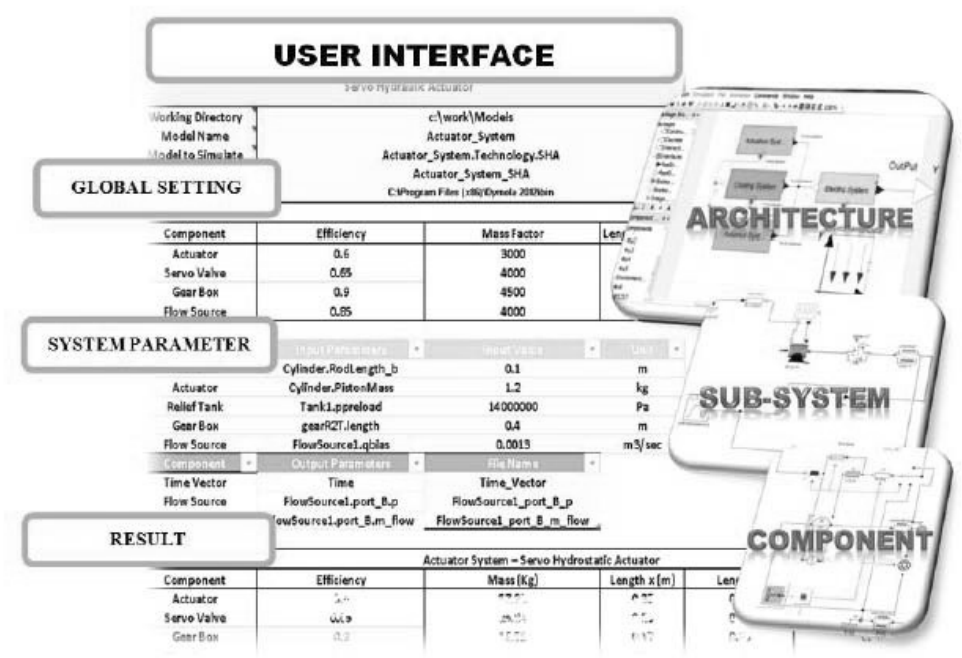

Figure 4-1: Screenshot of CAVE user interface.

CAVE consists of a number of spreadsheets, each linked to a specific subsystem. The number of active spreadsheets is based on the provided hierarchical system decomposition (HSD). The spreadsheets act as a user interface between the CE and the dynamic models of subsystem where the CE controls the models by inputting the desired parameters, setting up and starting the simulation, and extracting and sorting the results. A specific spreadsheet is designed for system architecting where various system architectures can be evaluated. A sample spreadsheet from the CAVE framework is showed in figures 4-1. An example of extracted simulation results of the power consumed by various cooling technologies over a predefined flight profile is shown in figure 4-2.

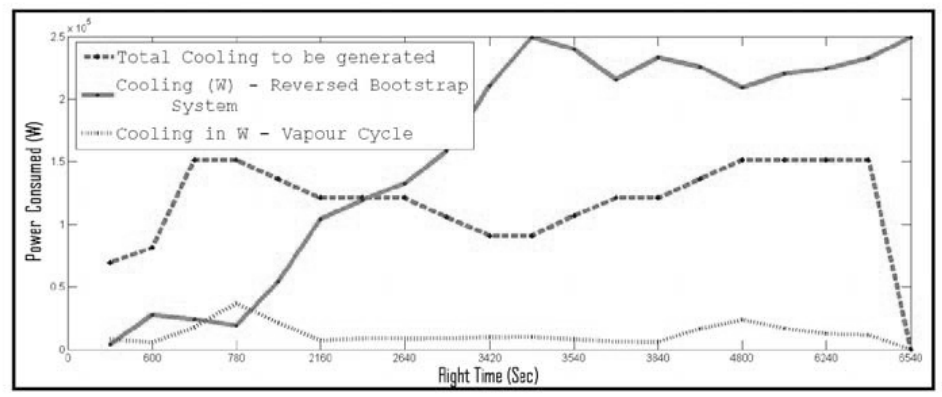

Figure 4-2: Sample result of CAVE, comparing cooling power produced by reversed bootstrap and vapour cycle system. 


\subsection{Paper II: Collaborative Multidisciplinary Design Optimization: A Framework Applied on Aircraft Conceptual System Design}

Integration of HFMs into conceptual design requires the knowledge of domain experts, who are the ones who create and develop such models. To integrate domain expert into the design process efficiently, a collaborative multidisciplinary design optimization (CMDO) framework, including the definition of roles and tasks, is first introduced in this paper, see figure $3-1$.

Optimization is shown to be an important tool to search the expanded design space in the presence of HFMs and optimize new concepts early in the conceptual phase. Although CAVE has a multidisciplinary character, it is limited to dynamic system simulation. Hence, CAVE is used within an MDO process that includes CAD and aerodynamic simulation to represent a product more precisely, see figure 4.3. CMDO has proven to be advantageous for managing the complexity of multidisciplinary design that includes different disciplines and associated domain experts. A case study from the aeronautic industry to design and optimize an aircraft actuation system architecture to operate the aircraft's flaps is presented. The objectives are to minimize power consumption and the size of the system with respect to a predefined flight profile and constraint on the wing geometry.

The results have proven the capability of CAVE to work efficiently with other design disciplines and the advantages of bringing optimization into the conceptual phase. The optimization is also performed with satisfactory speed and accuracy (in presence of surrogate models), which are vital factors in a conceptual study and in particular in presence of HFMs.

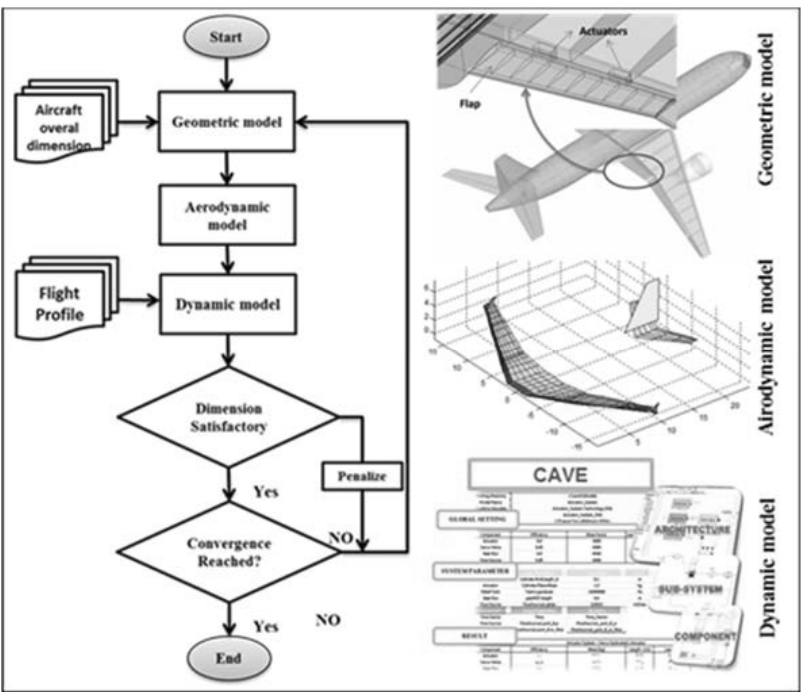

Figure 4-3: MDO framework including CAVE. 


\subsection{Paper III: Implementation of Collaborative MDO for Conceptual Design of a Complex Engineering Product}

The CMDO framework is used in this paper to optimize an innovative concept for a tidal power plant called Deep Green. Deep Green is a complex product that includes various tightly coupled disciplines. The project was developed within a large engineering design team. The project comprises two iterations. In the first iteration, the developed CMDO framework (presented in paper II) is used to design and optimize the concept. The technical and collaborative challenges of the implementation have been investigated and modifications made to the defined roles, tasks, and collaborative processes to improve the framework, see figure 4-4. In the second iteration, the modified CMDO framework is used to design and optimize the same concept.
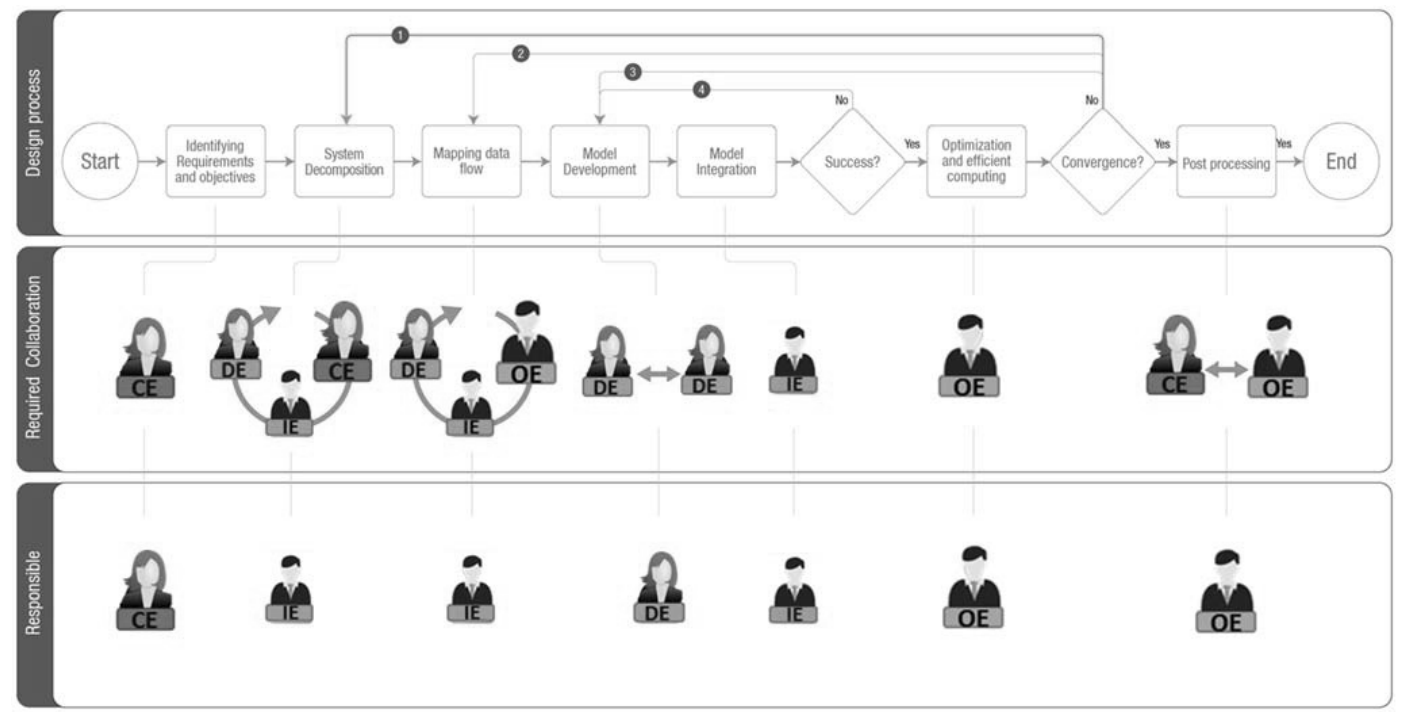

Figure 4-4: Modified CMDO framework.

A significant reduction in time spent performing the CMDO tasks is observed in iteration II due to fewer technical and collaboration challenges, see figure 4-5. Moreover, the number of feasible design points and the accuracy of the results are increased considerably compared to iteration I, see figure 3-9. 


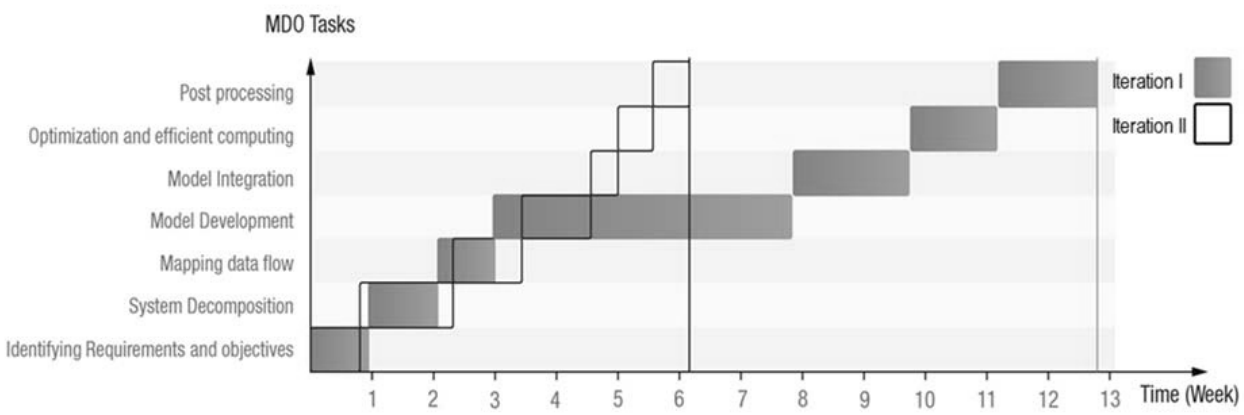

Figure 4-5: Time to perform each task during the course of the study. Iterations I and II show the implementation time of the old and the modified CMDO.

\subsection{Paper IV: A Guideline to Facilitate the Implementation of Collaborative MDO in Conceptual Design of Complex Products}

One important aspect of any research is the generality of the proposed method. For this reason, in this paper CMDO is used to design and optimize various products in order to evaluate the capability and generality of the proposed framework. The concept of CMDO has been proposed to graduate students on a project course developed at Linköping University. On the course students with different engineering backgrounds are grouped into a number of design teams to solve different MDO problems. The team members are instructed to follow the CMDO framework outlined in paper III. The implementation challenges are investigated from both technical and collaboration perspectives with the help of a survey consisting of interviews with team members and questionnaires on the framework, tasks, and processes. The results of the survey are restructured and used to modify the CMDO framework.

Finally, the results of this paper are restructured and summarized in the form of a stepby-step guideline that can be used by MDO practitioners to facilitate the implementation of CMDO within a large engineering team. The guideline has been extensively described in chapter 3.2 and an overview of the guideline is shown in figure 3.2. The guideline is made up of 4 main blocks containing various technical and collaborative tasks, which are presented in section 3.2 and figures 3-2 to 3-6. 


\subsection{Paper V: A Comprehensive Computational MDO Approach for a Tidal Power Plant Turbine}

Paper $[\mathrm{V}]$ provides more detailed information about the technical implementation of the CMDO framework as regards design and optimization of the Deep Green concept presented in paper III. In fact, the technical details regarding problem formulation, disciplinary modeling (including CAD, CFD, FEM and dynamic modeling), model integration and optimization are discussed extensively in this paper. The paper also highlights the high fidelity level of the models and the precision of the optimization results, see figure 4-6.

The paper also investigates the effect of relational and non-relational parameterization techniques on the robustness and flexibility of the conceptual design. The inherent properties of the relational design parameterization contribute to an efficient parametric modeling with improved communication between different disciplines. This enhances the performance of the MDO process and allows a more flexible and robust design.

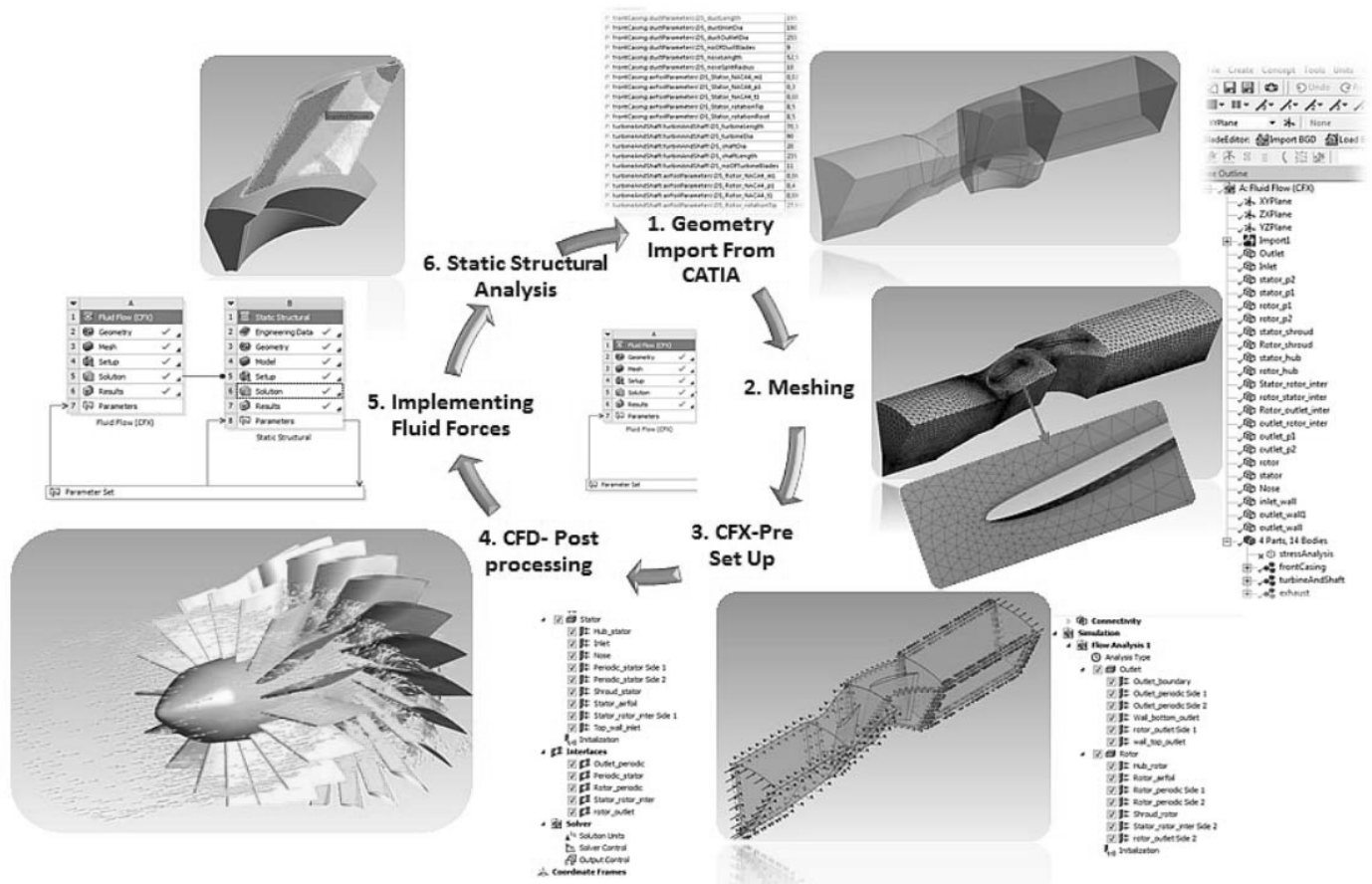

Figure 4-6: Computational fluid and structural analysis of the turbine' system of the Deep Green concept. 


\subsection{Paper VI: Rapid Concept Realization for Conceptual Design of Modular Industrial Robots}

Validation and verification of the high fidelity models in conceptual design is a hard task due to the lack of empirical data regarding the novel concepts under evaluation. This paper shows how a physical prototype can be used to enable model validation and illuminate unknown-unknown parts of the design, the strengths and shortcomings of a design and the manufacturability of the concept, and boost the engineering know-how regarding the performance of the concept. Simplification is a fundamental and inseparable part of conceptual design. High fidelity models should also be simplified in order to be used efficiently in conceptual design. Simplification should, however, be done logically in order not to extinguish the critical information about the concept under development. This paper shows how such simplified models can be used to provide higher fidelity analysis and even illuminate the unknown-unknown parts of the design by constructing a physical demonstrator, which is impossible to achieve with typical conceptual models.

In this study, the HFMs are developed for the conceptual design of a modular industrial robot. They are also used to build and control a down-scaled prototype. CAD and dynamic simulation are two important high fidelity tools in this study. A simplified dynamic system simulation is developed to provide information that is useful in the conceptual analysis such as cycle time and trajectory mapping. An example of the developed models and the physical prototype is shown in figures 4-7.

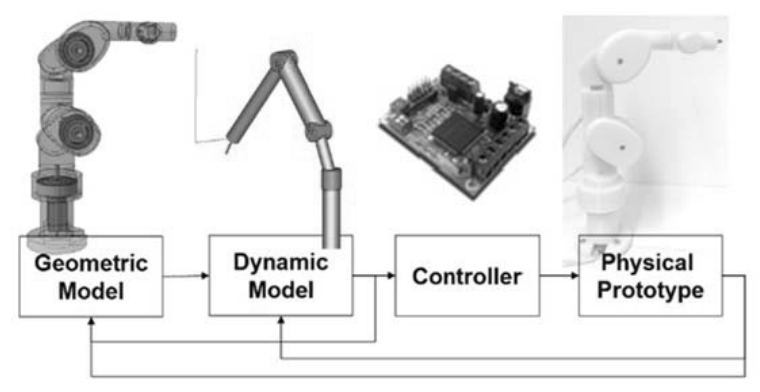

Figure 4-7: The process of concept realization of the physical prototype of an industrial robot.

Even though paper [VI] does not contain any actual MDO, it shows how HFMs can be used in conceptual design and thus constitutes the starting point for this research and is an important part of the background for developing the CMDO framework. Examples of the results provided by the physical prototype to validate the performance of HFMs are shown in figure $4-8$. 
46 Summary of the appended papers

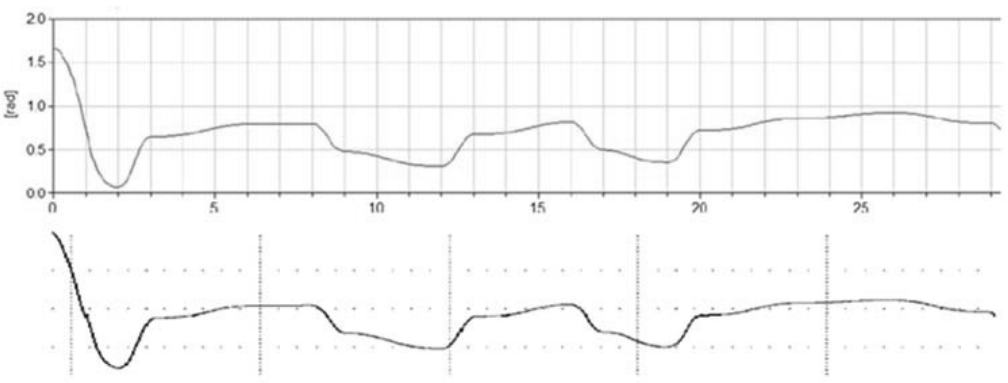

Figure 4-8: Comparison of the performed angular movement of the 2nd axis of the robot with the dynamic model (top) and the physical prototype (bottom). 


\section{5}

\section{Discussion}

$\mathrm{I}$

NTEGRATION of higher fidelity models (HFMs) and MDO in conceptual design is the main focus of this thesis. HFMs have been used in industry for decades and their effectiveness to increase the knowledge regarding the product is no longer questioned. However, they are typically used in later stages of the design process due to expertise and information being required to develop them. On the other hand, MDO has proven to be useful to efficiently search the design space and find optimal designs. MDO has also been developed substantially over the past three decades but is still not widely used in industry due to the many barriers to its implementation.

This work proposes some methods and tools to help industry, efficiently bring HFMs and MDO into the conceptual design process. However, the methods have been developed under certain assumptions and limitations and a discussion is therefore required regarding how the methods have been developed and how they could be expanded to be easily employed in industry. Moreover, in a broader scope, design research should be verified to provide generic and trustable results. A discussion of the generality of the research findings is therefore presented at the end of this chapter.

\subsection{Collaborative Aircraft Vehicle Engineering (CAVE) and the idea of CMDO}

As mentioned earlier, novel design tools are required to integrate HFMs into the conceptual design efficiently. CAVE has therefore been developed to facilitate this integration by enabling hierarchical system decomposition and collaborative design. CAVE as a design tool (presented in paper I) is limited to one specific discipline (dynamic system simulation) and the application of aircraft system design. In fact, CAVE provides an 
environment for domain experts to develop different aircraft subsystems independently and integrate them virtually to create a complete system. Conceptual engineers, on the other hand, can use the tool to evaluate various system architectures by changing the design parameters or entire subsystems that have been developed and integrated into the tool. CAVE has been used to show the importance of collaboration between the domain experts and conceptual engineers to increase the knowledge regarding the concept under evaluation. CAVE as a concept, however, is generic and expandable to multi-domains and various applications. In order to expand CAVE efficiently, however, the following hypotheses need to be taken into account:

- Domain experts are well-capable of developing or modifying HFMs for use in CD

- Collaboration between CEs and DEs is a necessity in order to define the design requirements

- Simplification of problems using hierarchical system decomposition is critical to efficiently develop the HFMs

- The user friendliness of the tool is a vital factor which is highly appreciated by CEs

- A modular design approach has proven to be useful as a facilitator of model development and design collaboration

In order to bring multi-dimensional analysis into conceptual design and boost the knowledge foundation, CAVE needs to work with other design tools. Hence, an MDO framework has been developed to search the design space, see paper II. In order to perform the MDO efficiently in conceptual design, however, specific expertise and a novel collaboration process between the experts are required. A highly flexible collaborative design framework (CMDO) is thus proposed as a key enabler to manage both the technical and collaborative complexities. In fact, the CMDO framework has been developed in this thesis to bring out the advantages of collaborative design and manage the complexity of implementing MDO in CD. The idea of CMDO is taken from the basic principles used to develop CAVE, which is to bring domain experts' knowledge into conceptual design through collaborative design. The basic elements of CMDO are thus various domain experts, redefined as roles, and the specific design processes that the experts should follow to manage the technical and collaborative challenges. These are the elements that constitute the definition of the CMDO framework. This makes the structure of CMDO framework different from classical MDO frameworks reported in the literature, where the focus is mainly on technical solutions to a specific problem without considering the required expertise and collaborative activities. 


\subsection{CMDO and challenges to its implementation}

CMDO has proven to be useful to facilitate the implementation of MDO, including HFMs in conceptual design, as shown in the appended papers II, III, IV and V. However, the efficient implementation of a CMDO framework within a team requires a structured process. Without such a process, the implementation may face many technical and nontechnical challenges. Some of these challenges, together with suggestions for avoiding or circumventing them, are discussed in this section, listed as follows:

- What are the important tasks involved in performing MDO in CD?

- Which expertise and knowledge are required to perform the tasks?

- How should different domain experts collaborate to perform the tasks efficiently?

- What are the different steps involved in implementing MDO efficiently?

- How can the performance of various MDO tasks be assessed?

In addition, the industrial use of MDO with HFMs is another ambitious goal of this study. However, a lack of proper MDO knowledge on the part of engineers in industry regarding MDO's potential, capabilities and methods for technical implementation is stated as the main source of the above-mentioned challenges (Hoogreef and La Rocca, 2015; Simpson and Martins, 2012). One remedy is apparently to offer these engineers training in MDO, which is a costly and time-consuming process. Another remedy is to provide a platform for engineers in industry to increase their experiential knowledge of MDO implementation. A step-by-step guideline is therefore presented in this thesis to assist MDO practitioners to efficiently implement CMDO within engineering teams and avoid the mentioned challenges.

The guideline has three functionalities:

- To advise on required collaboration among the team, including assigning roles and responsibilities to the team members

- To suggest the fundamental MDO tasks to be performed by team members

- To propose various assessment techniques to evaluate the performance of different elements of the framework from both collaborative and technical design aspects.

CMDO frameworks have been successfully implemented in different design applications and within various design teams. The common denominators of all applications include a focus on complex products, the conceptual design phase, high fidelity models, and a multidisciplinary team. The CMDO framework, including tasks and roles (conceptual engineer, interface expert, domain expert and optimization expert), is intended to be generic and independent of problem type and size, group expertise, implementation environment (industry or academia), design tools, etc. It is therefore crucial to evaluate the framework for applications that are as close as possible to the real products. The 
CMDO framework has been used in various applications from different domains such as aeronautics, robotics, underwater generators, and wind turbines, which can all be considered to be representative of complex products. The variety of the applications, problems and model fidelity presented in this thesis shows the high level of generality and adeptness of the CMDO framework. However, the CMDO framework has been developed in an academic setting with its natural limitations and the effect of such limitations on the generality of the framework therefore needs to be discussed.

\subsection{Generalization and industrial use of CMDO}

The generalization of the CMDO is studied by investigating its limitations and the assumptions made during its development, which are discussed in this section. This part is structured into team and collaboration and technical aspects of implementation.

\subsubsection{Team and collaboration}

In this study, the CMDO framework has been developed for use in conceptual design, where the objectives of the study are defined. Conceptual Engineers are therefore well capable of identifying the objectives. Another main task in the framework is model development, which requires expertise. Domain Experts are for this reason well suited to take responsibility for performing the modeling task. Coupling of the developed model to create the multidisciplinary system is an essential part of an MDO process and needs expertise and extensive experience. The Interface Expert role is therefore defined for an experienced engineer to facilitate the coupling process. Finally, another fundamental task in an MDO framework is optimization, where the problem is formulated and solved using analytical or numerical methods and where the results are visualized and analyzed. Optimization Expert is therefore defined as the role to deal with the optimization tasks. The general idea to define the tasks is derived from the classical tasks involved in an MDO framework. The tasks and the roles are therefore defined generically and independently of academia or industry. Hence, the responsibilities could also be efficiently assigned in industry as done in some of the case studies presented in the appended papers.

This research has been conducted in academia, where mainly $\mathrm{PhD}$ - and master-students have developed and evaluated the presented methods. Students are not expected to be as focused and experienced as experts in industry as regards the product under development. But they are typically highly familiar with state-of-the-art technologies regarding MDO techniques, design optimization, and novel model development techniques, e.g. modular and parametric modeling. Hence, the students are well suited to take the domain expert and optimization expert roles in a CMDO framework. On the other hand, familiarity with 
and long experience of the products and customer requirements are considered to be fundamental skills to take a wise role as a conceptual engineer and an interface expert. These roles therefore fit the experienced experts in industry well. The CMDO framework presented here has been developed in academia in close collaboration with industrial partners to leverage the benefits and potential of using the strengths of both academia and industry.

Companies are normally structured into various design departments and divisions, each responsible for specific parts of the design. Communication within a large organization requires specific procedures and perhaps also specific tools (Lundin et al., 2014). For example, PLM systems are nowadays widely used by large companies to facilitate the complex communications such as sharing models between two divisions. However, CMDO has been developed within an academic team where communication is assumed to be easier than at large companies. The groups' size in various applications was limited (max. 10 individuals and for many applications fewer), which is assumed to represent an MDO team in small or medium size companies. The framework should work efficiently for MDO groups of the same size in industry. Furthermore, as MDO is not frequently used in industry (at least not in conceptual design), the natural way to start using MDO is with a modest group size and hence the proposed framework should fit well. Larger groups, however, may need to adjust the framework, for example by identifying additional responsibilities or tasks mainly to control the collaborative activities and manage the group.

\subsubsection{Technical aspects}

Commercial design tools are used in all the case studies presented in this thesis, mainly due to their wide application in industry. Some companies, however, still prefer to develop and use their own in-house tools. The CMDO framework is generic and should not be dependent on the tools used. This has been proven by using different design tools in a number of the case studies presented in the appended papers. Nevertheless, using some of the techniques presented in the theoretical background, such as surrogate modeling or using functional mockup interface (FMI), could facilitate dealing with customized tools as well. Moreover, these techniques can also be used to reduce the number of occupied licenses for the disciplinary tools during the optimization process, as licenses are normally few and costly in industry. Computational time-efficient analysis is considered another advantages of the mentioned techniques. The downside of using such techniques, however, is of course not having absolute accuracy in the model outputs.

The models studied in this thesis are considered to be black box models with complex behavior (non-linear) where the derivatives of the objectives and constraints cannot be found analytically. This is not an inappropriate assumption because problems in industry 
normally have similar properties such as complex, not convex, and non-linear models. The focus in the thesis is therefore on non-gradient-based optimization techniques. As these optimization methods are more generic compared to gradient-based techniques, the proposed methods should be applicable in a broad range of applications.

Many problems in industry are normally complex and characterized by a large number of variables, objectives and constraints, and where the solutions' robustness is important. This thesis, however, deals with simplified conceptual problems with a limited number of variables. This is mainly because real problems in the conceptual design phase often have these characteristic, as they are simplified to be solvable within a reasonable timeframe. Moreover, the first recommendation for industry to apply MDO in conceptual design is to "start simple" and then add details incrementally as the process progresses. It should also be considered that it is not realistic to tackle the hardest and biggest problems early in the design process and the proposed CMDO framework should thus be well suited to address the problems faced when introducing MDO in the conceptual design phase.

There are many technical aspects that are important when developing an MDO framework that have not been in focus in this thesis. Optimization algorithms, sensitivity analysis, sampling methods, meta-modeling techniques, dealing with uncertainties and postprocessing techniques are a few examples of such activities. This thesis is intended to provide a generic method independent of problem type and the tools used. This is the main reason not to consider the mentioned aspects in the proposed framework. Extra effort is therefore required to handle the specific technical issues raised in each specific case study. Another reason is the availability of the resources and extensive studies performed in optimization's community regarding the topics mentioned (Nocedal and Wright, 2006; Jurecka, 2007; Ben-Tal et al., 2009; Sobieszczanski-Sobieski et al., 2015; Koziel and Yang, 2011). However, the massive development in this area does not necessary mean that further development is unimportant but it highlights a need to change the direction of studies toward practical use of the techniques. The recommendation to industrial practitioners is to become familiar with the methods, tools, and algorithms that work well in their specific applications.

The model development and optimizations performed in the thesis are implemented using a standard PC with 32 GB RAM and a $3.2 \mathrm{GHz}$ processor. Typically, the optimizations in this thesis using real models did not consume more wall-clock time than 4-6 days (depending on the problem's size). This time was much reduced (from days to hours) for the same problem when meta-models were used, proving that industry does not need to make massive investments in hardware to implement CMDO in their design process. 


\subsection{Verification of the results}

As stated in Section 1.5.1, verification by means of acceptance by the research community and experienced engineers in the field is used to evaluate the validity of the methods developed in this thesis. To that end, the CAVE and CMDO frameworks have been developed in close collaboration with industry. The industrial partners have confirmed that the proposed method would be useful in improving their level of knowledge in conceptual design. The CMDO framework has also been developed and evaluated with the intention of answering real design problems raised by the industrial partners.

In addition, the results presented in papers I, II, III, and VI are verified through the double-blind peer review in the publication process, where specialists in the research area (reviewers and editors) evaluate the quality of the results. The results in papers IV and $\mathrm{V}$, which have been submitted for publication, have been verified by industrial partners and discussed with experts in the fields prior to submission. Furthermore, the performance of the CMDO framework developed in papers II, III, and IV has been evaluated and tested by peers in terms of students in various application studies. The validity of the results has thus been verified through acceptance by both the academic community and experts from industry.

Moreover, the results of HFMs and optimization also need to be confirmed to prove their effectiveness to improve the knowledge and provide verified results. This is even more critical in the conceptual design of unconventional products where the empirical data is not accessible and should ideally be confirmed by means of physical experiments. Rapid concept realization as proposed in paper VI has proven to be useful for different purposes, for example:

- To confirm the results obtained from HFMs and optimization

- To illuminate the unknown-unknown part of the design, which may be neglected in modeling phase

- To provide engineering understanding regarding the performance of the final product in the conceptual phase 



\section{6 \\ Conclusions}

$\mathrm{H}$

IGH fidelity models (HFMs) have high capabilities to provide valuable information regarding the design concepts under study. These are normally used in later stages of the design process (e.g. the detail design phase) where more information and expertise to develop them are accessible. This thesis has shown that HFMs could also be used in conceptual design to not only provide more analytical information regarding the concept under development but also to be used to develop functional physical prototypes that could raise the information level to a large extent. Integration of HFMs into conceptual design faces some difficulties and challenges such as extension of the design space and high demands for expertise to develop and deal with such detail models in the conceptual stage.

MDO has proven to be useful to search the design space in an efficient and structured way and furthermore to suggest optimal concepts. However, the implementation of MDO in conceptual design, and especially when using higher fidelity models, requires close collaboration between various experts. The CMDO framework has therefore been presented in this thesis to facilitate the implementation of MDO in conceptual design. The CMDO framework consists of defined roles and tasks and technical and collaborative processes to perform the tasks. The CMDO framework is based upon a solid foundation of case studies and has been evaluated and improved using various applications.

A step-by-step CMDO guideline is presented to MDO practitioners in industry to facilitate the implementation process. The guideline consists of four blocks, each providing detailed information regarding: object identification, hierarchical decomposition, mapping of the data flow, model development and model integration, and finally optimization and efficient computing. 
The CMDO guideline is expected to bring the MDO community one step closer to exploiting the full potential of MDO in industrial settings, as discussed in the thesis and which includes:

- Holistic system optimization

- The use of HFMs and refined analyses in conceptual design

- Efficient computing

- Reduction of complexity when dealing with coupled variables

- Improved communication between different design teams

- Allowing conceptual creativity while using HFMs in the design process

- Data visualization in multiple dimensions

\subsection{Answers to the Research Questions}

This section provides a summary of the answers to the research questions stated in Section 1.4 .

RQ1: How can the usage of High Fidelity Models be supported in the conceptual phase?

Collaboration between conceptual engineers and domain experts is a key factor to answer this question. Collaboration is required on different levels, such as problem identification and formulation, models development, model integration, etc. A software tool called CAVE (presented extensively in paper [I]) has been presented to facilitate the collaborative design technically. In fact, CAVE provides a platform that is accessible for both conceptual engineers and domain experts. Domain experts develop the models and integrate them into CAVE. The conceptual engineers could use CAVE to easily set up simulations, execute them, and extract the results through the CAVE interface without directly dealing with high fidelity tools. Although CAVE was developed as a specific tool for aircraft vehicle engineering, it showed that it was possible to develop such a framework that became an efficient way of working when connected to an MDO framework. The CMDO concept was thus further developed as an efficient tool to support conceptual design using HFMs. Dealing with HFMs in an MDO setting requires special expertise. Hence, a collaborative process has been defined to facilitate the required tasks. These are fully described in the CMDO framework presented in this thesis. It has then been shown in multiple applications that CMDO offers an efficient approach to integrate HFMs in conceptual design. 
RQ2: What are the important technical and non-technical barriers to implement $M D O$ in an engineering team and how can they be managed?

The technical and non-technical barriers to MDO implementation have been addressed by many researchers and MDO practitioners (Simpson and Martins, 2011; Belie, 2002; Agte et al., 2009; Sobieszczanski-sobieski et al., 2015). These barriers can be categorized as shown below:

- Complexity of collaborative design (relationships between individuals and workflow)

- MDO enabled modeling and simulation strategy

- Coupling and model integration in complex engineering systems

- Models and system fidelity, validations/verification, and dealing with uncertainties

- Concurrent, efficient computing

- Problem formulation and optimization

This thesis experiences many of the barriers mentioned above in various design applications. However, one of the main barriers to MDO implementation in industry is the lack of MDO expertise, support, and knowledge of how it should be implemented. Various roles are therefore defined to facilitate collaboration and support the conceptual engineer. CMDO is proposed with the intention of facilitating collaboration between the proposed roles and perform well-defined tasks. CMDO could contribute extensively from a practical perspective by facilitating:

- Collaboration between roles (e.g. between different experts)

- Model development with insight from MDO (e.g. parametric modeling)

- Model integration and assessment process

- Utilization of efficient computing early when developing the MDO framework

- Problem formulation and choosing an appropriate optimization strategy

RQ3: How can efficient implementation of an MDO framework in an engineering team be supported?

Efficient implementation has a broad meaning. However, two important factors have been recognized in this study. The most important factor is fewer implementation challenges or less effort required to set up an MDO framework. The other important factor is the inclusion of HFMs in order to increase knowledge and thus the efficiency of MDO. A CMDO guideline is presented in this thesis to support MDO practitioners with the intention of reducing implementation challenges of MDO using HFMs. The guideline consists of four blocks where each block suggests various tasks and collaborative processes 
to accomplish the tasks. The guideline supports the MDO practitioner with practical suggestions at various levels of a MDO process such as:

- Object identification

- Problem formulation, hierarchical system decomposition, and mapping the data flow

- Model development and assessment of the performance of the disciplinary models

- Model integration and evaluation of the performance of coupled models

- Suggestions for efficient computing and choosing optimization strategy

- Post-processing of the optimization results

\subsection{Future work}

The CMDO framework and the implementation guideline proposed in this thesis have been developed in academia using a limited number of case studies and applications. The ambitious goal of CMDO is to reduce product development cost and time. This is a hard task without evaluating the framework directly in industry. The first proposal is therefore to use and evaluate the CMDO framework in real industrial development processes and report the performance of the framework and its effects on design time, quality, and cost. Engineering teams in industry could provide valuable feedback for further improvements to the framework, e.g. regarding how efficiently they could follow the suggested technical and collaborative tasks and what the main challenges of implementation are. This would help to improve the CMDO guideline and hopefully bring us one step closer to preparing an CMDO handbook.

CMDO is presented as a framework intended for practical use in industry. However, there are many more practical issues that need to be considered when applying MDO to solve real-world problems. Examples of such practical issues include model validation and verification, uncertainty management, MDO architecture, optimization formulation and optimization algorithms as well as efficient computing methods. Further studies to address these issues are all suitable directions for future work.

Finally, the physical demonstrators have proven to be valuable tools to illuminate the unknown-unknown part of the design, validate the HFMs, confirm the optimization results, and provide an engineering understanding regarding the final concept early in the design process. Today, with the advancement of additive manufacturing, the development of physical prototypes seems even more straightforward. Further investigation of the use of physical demonstrators to improve conceptual design and validate the use of HFMs could also be a valuable direction for further research. 


\section{References}

Agte, J., de Weck, O., Sobieszczanski-Sobieski, J., Arendsen, P., Morris, A., and Spieck, M., "MDO: assessment and direction for advancement - an opinion of one international group," Structural and Multidisciplinary Optimization, Vol. 40, No. 1-6, 2010, pp. 17-33, 2009.

AIAA Technical Committee on MDO, "White Paper on Industrial Experience with MDO". American Institute of Aeronautics and Astronautics, 1998.

Amadori K., "Geometry Based Design Automaiton- Applied To Aircraft Modeling and Optimization", Linköping Studies in Science and Technology. Dessertation No. 1418, ISBN:978-91-7519-986-3, Linköping, Sweden, 2012.

ANSYS Mechanical, APDL Structural Analysis Guide, http://148.204.81.206/Ansys/150/ANSYS\%20Mechanical\%20APDL\%20Structural $\% 20$ Analysis\%20Guide.pdf, 2016.

Arora J., "Introduction to Optimum Design", 3rd Edition, ISBN-13: 978-0123813756, Academic Press, 2011.

Belie G., "Non-Techncial Barriers To Multidesciplinary Optimization In The Aerospace Industry", 9th AIAA/ISSMO Symposium on Multidisciplinary Analysis and Optimization. Atlanta, Georgia, USA, 2002.

Ben-Tal A., El Ghaoui L., Nemirovski A., "Robust Optimization", Princeton Series in Applied Mathematics, Princeton University Press, ISBN: 9780691143682, 2009.

Berard A., Rizzi A., Isikveren A.T., "A New Geometry Construction Tool for Aerospace Vehicle Pre-Design and Conceptual Design", ICAS 2008, September 2008, Anchorage, Alaska, USA. 2008.

Bhise V. D., "Designing Complex Products with Systems Engineering Processes and Techniques", ISBN 9781466507036, CRC Press, 2013.

Blessing L. T. M., Chakrabarti A., DRM, a Design Research Methodology, Springer, 2009.

Brandt S.A., Stiles R.J., Bertin J.J., Whitford R., "Introduction to Aeronautics: A Design Perspective", AIAA Educational Series, Reston, VA, USA, 1997

Braun R. and Kroo I., "Development and Application of the Collaborative Optimization Architecture in a Multidisciplinary Design Environment", Proceedings of the 
ICASE/NASA Langley Workshop on Multidisciplinary Design Optimization, Hampton, Virginia, March 1995.

Braun R., "Collaborative Optimization: an architecture for large-scale distributed design", PhD dissertation, Stanford University, Department of Aeronautics and Astronautics, May 1996.

Buur J., "A Theoretical Approach to Mechatronics Design". Doctoral Thesis, Thechnical University of Denmark, Lyngby, Denmark, 1990.

CD-adapco, Star-CCM+ User Guide. [Online] Available at: http://www.cdadapco.com/products/star-ccm\%C2\%AE/documentation , 2016.

Chapman C.B., Pinfold M., "The application of knowledge based engineering approach to rapid design and analysis od an automotive structure", Journal of Advances in Engineerign Software, Vol. 32, Issue 12, pp. 903-912, Dec. 2001.

Chawner J. R., Dannenhoffer J., Taylor N. J., "Geometry, Mesh Generation, and the CFD 2030 Vision", 46th AIAA Fluid Dynamics Conference AIAA Aviation, doi:10.2514/6.2016-3485, 2016.

Coello C. A., Van Veldhuizen D.A., and Lamont G. B., "Evolutionary Algorithms for Solving Multi-Objective Problems", Kluwer Academic Publishers, Boston, USA. 2002.

Cross N., "From a Design Science to a Design Discipline : Understanding Designerly Ways of Knowing and Thinking". In R. Michel, Design Reaserch Now-Essays and Selected Projects, pp. 41-45: Birkhäuser Basel, 2007.

Daintith J. and Wright E., "A Dictionary of Computing", 6th edition, ISBN: 9780199234004, Oxford University Press, 2008.

Danahy E., Hynes M., Schneider L., Dowling D., "The Aggregation Tool: Toward Collaborative Inquiry in Design-Based Science and Engineering Projects", ASEE Northeast Section Conference, University of Massachusetts Lowell, April 27-28, 2012.

Dassault system(1), CATIA V5, http://www.3ds.com/se/produkter-och-tjaenster/catia/, 2016.

Dassault $\quad$ system(2), Dymola, $\quad$ http://www.3ds.com/products services/catia/products/dymola, 2016.

Dassualt $\quad$ system(3), Solidwork, $\quad$ http://www.3ds.com/se/produkter-ochtjaenster/solidworks/, 2016.

Deb K., "Multiobjective Optimization Using Evolutionary Algorithms", John Wiley \& Sons, New York, NY, USA, 2001. 
Desjardin P. E., Bojko B. T., and McGurn M. T., " Initialization of high-order accuracy immersed interface CFD solvers using complex CAD geometry". Int. J. Numer. Meth. Engng, doi: 10.1002/nme.5294, 2016.

Dieter G. E., Kuhn H. A., Semiatin S. L., "Handbook of Workability and Process Design" University of Michigan, ISBN: 0871707780, ASM International, 9780871707789, 2003.

Du X., Chen W., "Sequential Optimization and Reliability Assessment Method for Efficient Probabilistic Design", ASME. J. Mech. Des. 2004;126(2):225-233. doi:10.1115/1.1649968, 2004.

Dugan U., "Solid Modeling and Applications: Rapid Prototyping, CAD and CAE Theory", Springer International Publishing, 10.1007/978-3-319-21822-9_8, http://dx.doi.org/10.1007/978-3-319-21822-9_8, P 223-286, 2016.

Eppinger S. D. "A planning method for integration of large-scale engineering systems." International Conference on Engineering Design. 1997.

Esteco, availale online in http://www.esteco.com/, 2016.

Eynard B., Liénard S., Charles S. and Odinot A., "Web-based Collaborative Engineering Support System: Applications in Mechanical Design and Structural Analysis" Concurrent Engineering June 2005 13: 145-153, doi:10.1177/1063293X05053799, 2005.

Fähling J., Leimeister J. M., \& Krcmar H., "Collaboration Engineering for Innovation Design Processes using the Outcome-driven approach", In: 44. Hawaii International Conference on System Sciences (HICSS) 2011, Kauai, Hawaii, USA, 2011.

Favela, J., Wong A., Chakravarthy A., "Supporting collaborative engineering design" Journal of Engineering with Computers, Springer, 9: 125. doi:10.1007/BF01206343, 1993.

Ferziger J. H., Peric M., "Computational Methods for Fluid Dynamics", 3rd Edition ISBN 978-3-642-56026-2, DOI 10.1007/978-3-642-56026-2, Springer-Verlag Berlin Heidelberg, 2002.

Fluent, A., ANSYS Fluent User Guide. [Online] Available at: http://aerojet.engr.ucdavis.edu/fluenthelp/html/ug/node155.htm, 2016.

Forrester A., Sobester A. and Keane A., "Engineering design via surrogate modeling: a practical guide", John WiLey and Sons, 2008.

Fritzson P., "Principle of object-Oriented Modeling and Simulation with Modelica 2.1", IEEE Press, Piscataway, New Jersey, 2004.

Functional Mock-up Interface, available online in www.fmi-standard.org , 2016 
Giesing JP, Barthelemy, L-FM, "A summary of industry MOD applications and needs", AIAA 1998-4737, AIAA/USAF/NASA/ISSMO Symposium on Multidisciplinary Analysis and Optimization, St. Louis, MO, USA, Sep.1998.

Goldberg D. E., "Genetic Algorithms in Search, Optimization, and Machine Learning" Addison-Wesley Publishing Company, 1989.

Haake J. M., Hussein T., Joop B., Lukosch S., Veiel D., and Ziegler J., "Modeling and exploiting context for adaptive collaboration", International Journal for Cooperative Information Systems (IJCIS), 19(1-2):71-120, 2010.

Hoogreef M. F. M., La Rocca G., "An MDO advisory system supported by knowledgebased technologies", 16th AIAA/ISSMO Multidisciplinary Analysis and Optimization Conference- AIAA Aviation, American Institute of Aeronautics and Astronautics, 2015.

Howard T., Culley S. and Dekoninck E., "Creativity In The Engineering Design Process", International Conference on Engineerign Design, ICED’07 28-31 Aug 2007, CITE Des Science ET DE L'Industrie, Paris, France, 2007.

Hwang C., Paidy S., and Yoon K., "Mathematical programming with multiple objectives: a tutorial," Computers \& Operations Research, vol. 7, pp. 5-31, 1980.

Hwang J.T., Martins J. R. R. A. "GeoMACH: Geometry-Centric MDAO of Aircraft Configurations with High Fidelity", Proceedings of the 14th AIAA/ISSMO Multidisciplinary Analysis Optimization Conference, Indianapolis, USA, 2012.

iSIGHT, available online in, http://www.3ds.com/productsservices/simulia/products/isight-simulia-execution-engine, 2016.

Jenkinson L.R., Simpkin P., Rhodes D., "Civil Jet Aircraft Design", ButterworthHeinemann Publications, Burlington, 1999.

Jones D. R., Schonlau M. and Welch W. J. , "Efficient Global Optimization of Expensive Black-Box Functions ", Journal of Global Optimization 13: 455-492, Kluwer Academic Publishers. 1998.

Jurecka F., "Robust design optimization based on metamodeling techniques". Aachen: Shaker, 2007.

Karlsson L., Löfstrand M., Larsson A., Larsson T., Törlind P., Elfström B. O., \& Isaksson, O., "Information driven collaborative engineering-enabing functional product innovation", In Challenges in Collaborative Engineering-CCE'05-the knowledge perspective in collaborative engineering. School of engineeering, 2005.

Klein M., Sayama H., Faratin P., Bar-Yam Y., Braha D., Minai A., Bar-Yam Y. "The dynamics of collaborative design: Insights from complex systems and negotiation research". Complex Engineered Systems: Science Meets Technology, Springer, New York, pp 158-174 Cros, 2006. 
Kodiyalam, S. and Sobieszczanski-Sobieski, J., "Multidisciplinary design optimization some formal methods, framework requirements, and application to vehicle design", Int. J. Vehicle Design (Special Issue), pp. 3-22, 2002.

Kolfschoten G.L. and De Vreede G., "The Collaboration Engineering Approach for Designing Collaboration Processes", Groupware: Design, Implementation, and Use, 13th International Workshop, CRIWG 2007, Bariloche, Argentina, September 1620, 2007.

Koziel S., Yang X. S., "Computational Optimization, Methods and Algorithms", Volume 356 of Studies in Computational Intelligence, Springer Springer Science \& Business Media, 2011.

Kroo I., Altus S., Braun R. Gage P. and Sobieszczanski-Sobieski J., "Multidisciplinary Optimization Methods for Aircraft Preliminary Design", AIAA Paper 94-4325-CP, 1994.

Krus P. and Ölvander J., "Performance index and meta-optimization of a direct search optimization method", Engineering optimization (Print), (45), 10, 1167-1185, 2013.

Krus P., Andersson J., "Optimizing optimization for design optimization", Proceeding of DETC , 2003, ASME Design Automation Conference, Chicago, Illinois, USA, 2003.

Kvan A., "Collaborative design: what is it? Automation in Construction", Elsevier July 2000.

La Rocca, G., Cerulli, C., Schut, J. E., Nawijn, M., Baluch, H. A., Berends, J., and Tooren, M. J. L. V., "Knowledge-Based Engineering supported design: Development of Design and Engineering Engines for multidisciplinary Design and Optimization", Tech. rep., TU Delft. 2011.

La Rocca, G., van Tooren, M.J.L., "Enabling distributed multi-disciplinary design of complex products: a knowledge based engineering approach", J. Design Research, Vol. 5, No. 3, pp. 333-352, 2007.

Larson W.J. and Wertz J.R., "Space Mission Analysis and Design" , Kluwer Academic Publ. and Microcosm Inc., 1999.

Ledermann C., Hanske C., Wenzel J., Ermanni P. \& Kelm R., "Associative Parametric CAE methods in the aircraft pre-design", Aerospace Science and Technology 9 (7) (2005) 641-651. 2005.

Lilienthal M., "Observations on the Uses of Modeling and Simulation", Defense Modeling and Simulation Office, presented to the Committee on Bridging Design and Manufacturing, National Research Council, Washington, D.C., February 24-25, 2003 .

Loch C.H., DeMeyer A. and Pich M.T., "Managing the Unknown: A New Approach to Managing High Uncertainty and Risk in Projects", NJ: John Wiley \& Sons, 2006. 
Lu S.C.Y. and Cai J., "A collaborative design process model in the sociotechnical engineering design framework". AI EDAM, 15, pp. 3-20, 2001.

Lukosch S., and Kolfschoten G. L. "Towards effective collaborative design and engineering", Paper presented at the 2d IUI Workshop on Semantic Models for Adaptive Interactive Systems, Palo Alto, CA, February 13-16, 2011.

Lundin M., Lejon E., Dagman A., Näsström M., \& Jeppsson P., "An Empirical Study of Information Exchange and Design Support in Product Family Development", In ASME 2014 International Design Engineering Technical Conferences and Computers and Information in Engineering Conference (pp. V01BT02A018V01BT02A018). American Society of Mechanical Engineers, 2014.

Martins J. R. R. A. and Lambe A. B., "Multidisciplinary design optimization: A Survey of architectures", AIAA Journal, 51(9), DOI: 10.2514/1.J051895, 2013.

Martins J.D., and Simpson T.W.., "A Method to Manage Uncertainty During SystemLevel Conceptual Design", ASE DETC2005-84984, ASME Design Engineering Technical Conference - Design Automation Conference, Long Beach, California, USA. September 24-28, 2005.

MathWorks, Simulink, http://se.mathworks.com/products/simulink/, 2016.

Mavris D. N., and De Laurentis D. A., "A probabilistic approach for examining aircraft concept feasibility and viability", Aircraft Design, 3 pp.79-101, 2000.

McKay M.D., Beckman R.J. and Conover W.J., "A Comparison of Three Methods for Selecting Values of Input Variables in the Analysis of Output from a Computer Code, Technometrics, Vol. 21. No. 2 , 1979, pp. 239-245, 1979.

Microflo, IES, https://www.iesve.com/software/ve-for-engineers/module/MicroFlo/463, 2016.

Minesto, available online in http://minesto.com/, 2016.

Modelica Association, "Modelica A Unified Object-Oriented Language for Physical Systems Modeling, Language Specification, http://www.modelica.org, 1999.

MSC software, Adams, http://www.mscsoftware.com/product/adams, 2016.

Myers R.H., Montgomery, DC and Anderson-Cook, CM, "Response Surface Methodology, Process and Product Optimization Using Designed Experiments", Third Version, John Wiley \& sons, Inc., Hoboken, New Jersey, USA, 2009.

Myers, R. H. and Montgomery, D. C., "Response Surface Methodology: Process and Product Optimization Using Designed Experiments", Wiley \& Sons, New York, 1995. 
Nocedal J., Wright S., "Numerical Optimization", Springer Series in Operations Research and Financial Engineering, Springer-Verlag New York, ISBN 978-0-387-30303-1, DOI 10.1007/978-0-387-40065-5, 2006.

Nosratollahi M., Mortazavi M., Adami A., Hosseini M., "Multidisciplinary design optimization of a reentry vehicle using genetic algorithm" Aircraft Engineering and Aerospace Technology 2010 82:3 , 194-203, 2010.

OpenMDAO, availale online in, http://openmdao.org/, 2016.

Oxford English Dictionary, avalilable online in, " http://www.oed.com/ ", 2016.

Pahl G., Beitz W., "Enginering Design-A systematic Approahch", Springer Verlag, London, 1996.

Pahl G., Beitz W., Feldhusen J., Grote K.H., "Engineering design - a systematic approach", 3rd edn. Springer, London. Translated and Edited by K Wallace, L Blessing, 2007.

Paternò F., "Towards a UML for Interactive Systems", In: 8th International Conference on Engineering for Human-Computer Interaction. Lectures Notes Computer Science 2001 .

Pebesma E. J., and Weeseling C.G., "GSTAT: A Program for Geostatistical Modeling, Prediction and Simulation", Computer \& Science Vol. 24, No. 1, pp. 17-31, 1998.

Peng C., "Exploring communication in collaborative design: co-operative architectural modeling" Journal of Computer-Supported Cooperative Work, 15 (1) (1994), pp. 19-44, 1994.

Persson J., "Design and Optimization under Uncertainties, A simulation and Surrogate Model Based Approach" Licentiate thesis, Linkoping University, 2012.

Persson J., "Efficient Optimization of Complex Products: A Simulation and Surrogate Model Based Approach", Linköping studies in Science and Technology. Dissertations,1655- 2015.

Pokojski, J., "Knowledge based engineering and intelligent personal assistant context in distributed design". In Intelligent Computing in Engineering and Architecture (pp. 519-528). Springer Berlin Heidelberg, 2006.

ProEngineer, CREO, PTC, http://www.ptc.com/cad/pro-engineer, 2016

Queipo N.V., Haftka R.T., Shyy W., Goel T., Vaidyanathan R., Tucker P.K., "Surrogatebased analysis and optimization", Progress in Aerospace Sciences, Elsevier January 2005 .

Raynould M.T., "Test and evolution of Complex System". John Wiley \& sons, 1996.

Red Cedar Technology, availale online in , http://www.redcedartech.com/, 2016. 
Reddy J., "An Introduction to the Finite Element Method" 3rd Edition, ISBN-13: 9780072466850, ISBN-10: 0072466855, McGraw-Hill Mechanical Engineering, 2005.

Riccardi A., "Multidisciplinary Design Optimization for Space Applications". Diss. Staatsund Universitätsbibliothek Bremen, 2012.

Robinson, S., "Conceptual Modelling for Simulation Part I: Definition and Requirements." Journal of the Operational Research Society 59 (3): 278-290, 2008..

Saltelli A., Chan K., and Scott E.M., "Sensitivity analysis". Wiley Series in Probability and Statistics. Wiley, 2000.

Saltelli, A., Ratto, M., Andres, T., Campolongo, F., Cariboni, J., Gatelli, D., Saisana, M., and Tarantola, S. Global Sensitivity Analysis. The Primer, John Wiley and Sons, 2008 .

Savic D., "Single-objective vs. multiobjective optimisation for integrated decision support." Integrated Assessment and Decision Support 1, pp: 7-12, 2002.

Simpson T. W. and Martins J. R. R. A., "Multidisciplinary design optimization for complex engineered systems design: Report from an NSF workshop". Journal of Mechanical Design, 133(10):101002, October 2011. doi:10.1115/1.4004465, 2011.

Simpson T. W., Mauery T. M., Korte J. J. and Mistree F., "Kriging models for global approximation in simulation-based multidisciplinary design optimization". In: AIAA journal 39.12, pp. 2233-2241, 2001.

Simulia, Dassualt $\quad$ system, $\quad \underline{\text { http://www.3ds.com/products- }}$ services/simulia/products/abaqus/, 2016

Sobieszczanski-Sobieski J., "Multidisciplinary Optimization for Engineering Systems: Achievements and Potential". NASA TM 101566, March 1989.

Sobieszczanski-Sobieski J., Agte J.S. and Sandusky R.R.Jr., Bi-Level Integrated System Synthesis (BLISS), NASA TM-1998-208715, August 1998.

Sobieszczanski-Sobieski J., Emiley M.S., Agte J.S. and Sandusky R.R.Jr., Advancement of Bi-Level Integrated System Synthesis (BLISS), NASA TM-2000-210305, December 2000 .

Sobieszczanski-Sobieski J., Morris A., Tooren M. V., "Multidisciplinary Design Optimization Supported by Knowledge Based Engineering", John Wiley and Sons, 2015 .

Sorcer, Sourcer Soft, available online in http://sorcersoft.com/sorcer/, 2016.

Steinkellner S., "Aircraft Vehicle Systems Modeling and Simulation under Uncertainty", Licentiate thesis, Linkoping University, 2011.

Steuer R., "Multiple criteria optimization: theory, computation and application", New York, John Wiley \& Sons, Inc., 1986. 
Sunnersjö S., Cederfeldt M., Elgh F., Rask I., "A transparent design system for iterative product development", Journal of Computing and Information Science in Engineering. 2006;6(3):300-7, 2006.

Tarkian M. "Design Automation for Multidisciplinary Optimization- A High Level CAD Template Approach" Linköping studies in Science and Technology. Dissertations,1479- 2012.

Tarkian M. "Design Reuse and Automation - On high Level CAD Modeling for Multidisciplinary Design and Optimization ", Licentiate thesis, Linkoping University, 2009.

Tarkian M., Persson J., Ölvander J., Feng X., "Multidisciplinary Design Optimization of Modular Industrial Robots by Utilizing High Level CAD Templates", ASME J. Mechanical Design 134(12), 124502, doi:10.1115/1.4007697, 2012

Törlind P., and Larsson A., "Support for Informal Communication in Distributed Engineering Design Teams", Annals of 2002 Int'l CIRP Design Seminar, 16-18 May in Hong Kong, 2002.

Uflacker M., Zeier A., "A semantic network approach to analyzing virtual team interactions in the early stages of conceptual design". Future Gener Comput Syst 27(1):88-99, 2011.

Ullman D. G., "The Mechanical Design Process". 3rd ed. McGraw-Hill Higher Education, New York, 2003.

Ulrich K. T., Eppinger S.D., "Product Design and Development", McGraw-Hill, New York, 6th edition 2016.

Vandenbrande J.H., Granline T.A., and Hogan T.," The search for the perfect body: Shape control for multidisciplinary design optimization," 44th Aerospace Sciences Meeting and Exhibit, Jan.2006, Reno, NV, USA, 2006.

Vasilopoulos I., Agarwal D., Meyer M., Robinson T. T., \& Armstrong C. G., "Linking Parametric CAD with Adjoint Surface Sensitivities". European Congress on Computational Methods in Applied Sciences and Engineering, Athens, Greece, 2016.

Wang L., Shen W., Xie H., Neelmakvil J., Pardasani A., "Collaborative Concetual DesignState of the Art and Future Trend", Journal of Computer-Aided Design, V. 34, pp. 981-996, 2002.

Welle, B., J. Haymaker, M. Fischer, and V. Bazjanac, "CAD-Centric Attribution Methodology for Multidisciplinary Optimization (CAMMO): Enabling Designers to Efficiently Formulate and Evaluate Large Design Spaces", Technical Report, Number:TR195, Center For Intergrated Facility Engineering, Stanford University, 2012. 
Woodbury R. "Elements of Parametric Design". Routledge, Taylor and rancis Group. ISBN 0415779871. 2010.

Woodbury R., Williamson S., Beesley P., "Parametric Modeling as a Design Representation in Architecture: a process account", Cumulative Index of Computer Aided Architectural Design, 2006.

Yi S.I., Shin J.K., Park G.J., Comparison of MDO methods with mathematical examples. Structural and Multidisciplinary Optimization, 35(5):391-402, 2008.

Zeyl D.J, "Timaeus by Plato, Translated with introduction, by Donald J. Zeyl", Hackett Publishing, 2000.

Zhang H., Wang H., Chen D., "To Implement Distributed Multidisciplinary Federated Simulation for Collaborative Product Development", Computer Supported Cooperative Work in Design IV: 11th International Conference, CSCWD 2007, Melbourne, Australia, 2007. 
APPENDED PAPERS 



\section{Appended Papers}

The articles associated with this thesis have been removed for copyright reasons. For more details about these see:

http://urn.kb.se/resolve?urn=urn:nbn:se:liu:diva-131792 